\title{
ON BOUNDED VARIATION AND ABSOLUTE CONTINUITY FOR PARAMETRIC REPRESENTATIONS OF CONTINUOUS SURFACES
}

\author{
BY \\ PAUL V. REICHELDERFER
}

INTRODUCTION

1. A continuous curve $C$ in $x y z$-space may be defined by

$$
C: \quad x=x(u), \quad y=y(u), \quad z=z(u), \quad a \leqq u \leqq b,
$$

where each of the functions $x(u), y(u), z(u)$ is continuous on the closed interval $[a, b]$. The following facts are known $\left(^{1}\right)$ (see Rado [3, chap. I]; Saks [1, chap. IV]).

(1) A necessary and sufficient condition that the length $L(C)$ of $C$ be finite is that each of the functions $x(u), y(u), z(u)$ be of bounded variation on $[a, b]$.

(2) If the length $L(C)$ is finite, then each of the derivatives $x^{\prime}(u), y^{\prime}(u)$, $z^{\prime}(u)$ exists almost everywhere in $[a, b]$, is summable on $[a, b]$, and

$$
L(C) \geqq \int_{a}^{b}\left[x^{\prime}(u)^{2}+y^{\prime}(u)^{2}+z^{\prime}(u)^{2}\right]^{1 / 2} d u .
$$

(3) A necessary and sufficient condition that the sign of equality hold in this relation is that each of the functions $x(u), y(u), z(u)$ be absolutely continuous on $[a, b]$.

2. A continuous surface $S$ in $x y z$-space may be defined by

$$
S: \quad x=x(u, v), \quad y=y(u, v), \quad z=z(u, v), \quad a \leqq u \leqq b, \quad c \leqq v \leqq d,
$$

where each of the functions $x(u, v), y(u, v), z(u, v)$, is continuous on the closed (two-dimensional) interval $[a, b ; c, d]$. How may the concepts for the area of the surface $S$ and for bounded variation and absolute continuity of the representation of $S$ be defined so that theorems analogous to those for continuous curves cited in $\left.{ }^{2}\right) 1$ hold? For the special case in which $S$ may be given by relations of the form

$$
S: \quad x=u, \quad y=v, \quad z=f(u, v), \quad a \leqq u \leqq b, \quad c \leqq v \leqq d,
$$

Presented to the Society, April 17, 1942; received by the editors June 11, 1942.

(1) Numbers in square brackets refer to papers listed in the bibliography at the end of this paper.

(2) The notation $I, 6,2$, for example, refers to chapter I, section 6 , relation 2 in this paper When no chapter reference is given, the introduction is meant. 
Geöcze and Tonelli have shown a complete answer to this question (see Geöcze [1], Tonelli [1]). But for the general case, no satisfactory answer seems to be known.

3. It is the chief purpose of this paper to give an answer to the question just raised. In so doing, interesting generalizations and extensions of results in the literature will be obtained. Briefly, the program for procedure is the following. First, a definition for a continuous surface is made precise (see I, 1). Now concepts for bounded variation and absolute continuity of a representation for a curve are phrased in terms of the corresponding representations for the projections of this curve upon each of the coordinate axes. Here the definitions for bounded variation and absolute continuity of a representation for a surface will be made in terms of the corresponding representations for the projections of this surface upon each of the coordinate planes. For representations of the latter type, a hierarchy of definitions for bounded variation and absolute continuity is extant $\left(^{3}\right.$ ) (see $R^{2}[1]$ ). It will be desirable for the purpose of this paper to review these definitions, and to make certain additions to the theory developed in the work just cited (see II, III). Next, a definition for the area of a continuous surface will be given (see IV). This definition will be compared with that of the Geöcze area as defined by Rado (see IV, 17-20), and the Lebesgue area (see IV, 14-15); the latter area has been most frequently used in the literature. For the special case considered by Geöcze and Tonelli, it will be shown that the definitions advanced here are equivalent to those which they used (see IV, 16). With the definitions for the area of a surface, and for bounded variation and absolute continuity of its representations, thus formulated, it will be shown that theorems hold for continuous surfaces which are analogous to those cited in 1 for curves (see IV, 4, 6-13; V, 9-15). As an application of this theory, some of the results of Rado and Reichelderfer on convergence in area for surfaces (see $R^{2}$ [2]) will be generalized (see V).

4. For brevity, the following notations and conventions are adopted. The $u^{1} u^{2}$-plane will serve as a parameter plane; a point $\left(u^{1}, u^{2}\right)$ in it is denoted simply by $u$. The surfaces will be in $x^{1} x^{2} x^{3}$-space; a point $\left(x^{1}, x^{2}, x^{3}\right)$ in this space is denoted simply by $x$. With each point $x$, there is associated its projections on the respective coordinate planes given by

$$
{ }^{1} x=\left(0, x^{2}, x^{3}\right), \quad{ }^{2} x=\left(x^{1}, 0, x^{3}\right), \quad{ }^{3} x=\left(x^{1}, x^{2}, 0\right) .
$$

The planar exterior measure of any set $E$ in the $u$-plane is denoted by $|E|$. The set of interior points in the set $E$ is denoted by $E^{0}$.

If $a=\left(a^{1}, a^{2}, a^{3}\right)$ be any triple of real numbers, set

$$
|a|=\left(\left|a^{1}\right|,\left|a^{2}\right|,\left|a^{3}\right|\right), \quad\|a\|=\left[\left(a^{1}\right)^{2}+\left(a^{2}\right)^{2}+\left(a^{3}\right)^{2}\right]^{1 / 2} .
$$

A triple of real, finite, single-valued functions $x^{i}(u), i=1,2,3$, each defined

${ }^{(3)}$ The symbol $\mathrm{R}^{2}$ in this note is to be read "Rado and Reichelderfer." 
on a set $E$ in the $u$-plane is denoted by $[x(u), E]$, where $x(u)=\left(x^{1}(u), x^{2}(u)\right.$, $\left.x^{3}(u)\right)$ for $u=\left(u^{1}, u^{2}\right)$ in $E$. With each triple $[x(u), E]$ there are associated three triples $\left[{ }^{i} x(u), E\right]$ defined by

$$
\begin{array}{r}
{ }^{1} x(u)=\left(0, x^{2}(u), x^{3}(u)\right),{ }^{2} x(u)=\left(x^{1}(u), 0, x^{3}(u)\right),{ }^{3} x(u)=\left(x^{1}(u), x^{2}(u), 0\right), \\
u \text { in } E .
\end{array}
$$

A triple $[x(u), E]$ is said to possess any property which is possessed by each of the $x^{i}(u)$ for $i=1,2,3$ on the set $E$.

A two-dimensional interval in the $u$-plane is denoted generically by $I, \Im,[\alpha, \beta]$, or $\left[\alpha^{1}, \beta^{1} ; \alpha^{2}, \beta^{2}\right]$; it consists of all points $u$ satisfying $\alpha^{1} \leqq u^{1} \leqq \beta^{1}$, $\alpha^{2} \leqq u^{2} \leqq \beta^{2}$ where $\alpha^{1}<\beta^{1}, \alpha^{2}<\beta^{2}$. A connected open set in the $u$-plane is termed a domain, is denoted generically by $\mathcal{D}$ or $\mathfrak{D}$; if the boundary of a domain consists of a Jordan curve, then the closed connected set of points in the domain and on its boundary is termed a simple Jordan region, is denoted generically by $\beta, B$ or $\mathfrak{B}$. If the boundary of a domain consists of a finite number of Jordan curves, then the closed connected set of points in the domain and on its boundary is termed a Jordan region, is denoted generically by $\Re$. A sequence of domains $\mathcal{D}_{n}$ is said to fill up a domain $\mathcal{D}$ from the interior if each domain $\mathcal{D}_{n}$ is contained in $\mathcal{D}$, but for every closed set $F$ in $\mathcal{D}$ there exists an $n(F)$ such that $F$ is in $\mathcal{D}_{n}$ for every choice of $n$ exceeding $n(F)$. A sequence of Jordan regions $\Re_{n}$ fill up a Jordan region $\Re$ from the interior if their interiors $\Re_{n}^{0}$ fill up $\Re^{0}$ from the interior.

If $\mathfrak{B}$ is any simple Jordan region in the $u$-plane, then a finite system of nonoverlapping simple Jordan regions $B$ lying in $\mathfrak{B}$ is denoted generically by $S(\mathfrak{B})$. The maximum of the diameters of the domains $B$ in $S(\mathfrak{B})$ is denoted by $\|S(\mathfrak{B})\|$. If $\mathfrak{B}=\sum B$ for $B$ in $S(\mathfrak{B})$, then $S(\mathfrak{B})$ is termed a subdivision of $\mathfrak{B}$. In particular, if each of the simple Jordan regions $B$ in $S(\mathscr{B})$ is an interval, then $S(\mathfrak{B})$ is termed a finite interval system; if, moreover, $\mathfrak{B}$ is an interval and $S(\mathfrak{B})$ is a subdivision of $\mathfrak{B}$, then $S(\mathfrak{B})$ is termed an interval subdivision.

A $b$-function defined in $\mathfrak{B}$ is a law which associates with every simple Jordan region $B$ in $\mathfrak{B}$ a finite, real number $\phi(B)$; this function is denoted by $[\phi, \mathfrak{B}]$. This $b$-function is non-negative if $\phi(B)$ is non-negative for every $B$ in $(\mathfrak{B})$. For a finite system $S(B)$, where $B$ is any simple Jordan region in $\mathfrak{B}$, set

$$
\begin{aligned}
\phi(S(B)) & =\sum \phi(\beta) \text { for } \beta \text { in } S(B) ; \\
U(B ;[\phi, \mathfrak{B}]) & =\text { l.u.b. } \phi(S(B)) \text { for all finite systems } S(B) .
\end{aligned}
$$

Evidently $\phi(B) \leqq U(B ;[\phi, \mathfrak{B}]) \leqq U(\mathfrak{B} ;[\phi, \mathfrak{B}])$ for every choice of $B$ in $\mathfrak{B}$. Hence if $U(\mathfrak{B} ;[\phi, \mathfrak{B}])$ is finite, then $[U, \mathfrak{B}]$ is a $b$-function, and $[\phi, \mathfrak{B}]$ is said to possess a $U$-function. If $S(\mathfrak{B})$ is any finite system, then clearly $U(S(\mathfrak{B})$; $[\phi, \mathfrak{B}]) \leqq U(\mathfrak{B} ;[\phi, \mathfrak{B}])$.

5. If $\left[{ }^{i} \phi, \mathfrak{B}\right], i=1,2,3$, is a triple of $b$-functions having a common range of definition, set 


$$
\phi(B)=\left({ }^{1} \phi(B),{ }^{2} \phi(B),{ }^{3} \phi(B)\right), \quad \Phi(B)=\|\phi(B)\|, \quad \text { for } \quad B \text { in } \mathfrak{B} .
$$

If $[\phi, \mathfrak{B}]$ is a triple of non-negative $b$-functions, then clearly

$$
{ }^{i} \phi(B) \leqq \Phi(B) \leqq \sum_{i=1}^{3}{ }^{i} \phi(B) \quad \text { for } \quad B \text { in } \mathfrak{B} \text {. }
$$

Hence a necessary and sufficient condition that $[\Phi, \mathfrak{B}]$ have a $U$-function is that each member of the non-negative triple $[\phi, \mathfrak{B}]$ have a $U$-function, and

1. $U\left(B ;\left[{ }^{i} \phi, \mathfrak{B}\right]\right) \leqq U(B ;[\Phi, \mathfrak{B}]) \leqq \sum_{i=1}^{3} U\left(B ;\left[{ }^{i} \phi, \mathfrak{B}\right]\right)$ for $B$ in $\mathfrak{B}$.

Elementary considerations lead to the following

LemмA. Let $\left[\phi_{n}, \mathfrak{B}\right], n=0,1,2, \cdots$, be a sequence of triples of nonnegative $b$-functions for which $\lim \inf ^{i} \phi_{n}(B) \geqq{ }^{i} \phi_{0}(B)$ for $B$ in $\mathfrak{B}, i=1,2,3$. Then $\lim \inf \Phi_{n}(B) \geqq \Phi_{0}(B) ; \lim \inf U\left(B ;\left[{ }^{i} \phi_{n}, \mathfrak{B}\right]\right) \geqq U\left(B ;\left[{ }^{i} \phi_{0}, \mathfrak{B}\right]\right) ; \lim$ inf $U\left(B ;\left[\Phi_{n}, \mathfrak{B}\right] \geqq U\left(B ;\left[\Phi_{0}, \mathfrak{B}\right]\right)\right.$ for $B$ in $\mathfrak{B}$.

\section{Chapter I}

\section{ON CONTINUOUS SURFACES}

1. A definition for a continuous surface will now be recalled; since this definition is in the literature (see Rado [2, 3]), it will be merely sketched here for the convenience of the reader, and for the purpose of fixing notation in the sequel. Consider the class of all continuous triples $[x(u), \mathfrak{B}]$, where $\mathfrak{B}$ is a simple Jordan region in the $u$-plane. Let $\left[x_{1}(u), \mathfrak{B}_{1}\right],\left[x_{2}(u), \mathfrak{B}_{2}\right]$ be any two of these triples. Since $\mathfrak{B}_{1}$ and $\mathfrak{B}_{2}$ are simple Jordan regions, there exist topological maps of $\mathfrak{B}_{1}$ onto $\mathfrak{B}_{2}$ given by single-valued continuous pairs $\left[\bar{u}(u), \mathfrak{B}_{1}\right]$ having single-valued continuous inverses on $\mathfrak{B}_{2}$. Let $d(\bar{u})$ denote the maximum of $\left\|x_{1}(u)-x_{2}(\bar{u}(u))\right\|$ for $u$ in $\mathfrak{B}_{1}$. Put $d\left(\left[x_{1}, \mathfrak{B}_{1}\right],\left[x_{2}, \mathfrak{B}_{2}\right]\right)$ equal to the greatest lower bound of $d(\bar{u})$ for all topological maps $\left[\bar{u}(u), \mathfrak{B}_{1}\right]$ of $\mathfrak{B}_{1}$ onto $\mathfrak{B}_{2}$. It is easily verified that the binary relation $d$ thus defined in the class of all continuous triples has all the properties of a distance except one: the fact that $d\left(\left[x_{1}, \mathfrak{B}_{1}\right],\left[x_{2}, \mathfrak{B}_{2}\right]\right)$ is zero does not imply that $\mathfrak{B}_{1}$ and $\mathfrak{B}_{2}$ are identical and $x_{1}(u)=x_{2}(u)$ for $u$ in $\mathfrak{B}_{1} \cdot \mathfrak{B}_{2}$. In order to remedy this defect, one agrees that two of these triples $\left[x_{1}, \mathfrak{B}_{1}\right],\left[x_{2}, \mathfrak{B}_{2}\right]$ are in the $\sim$ relation provided $d\left(\left[x_{1}, \mathfrak{B}_{1}\right],\left[x_{2}, \mathfrak{B}_{2}\right]\right)=0$. It is readily verified that the binary relation $\sim$ is an equivalence relation; hence it partitions the class of all continuous triples into mutually exclusive sets of triples mutually in the $\sim$ relation; denote these sets generically by $S$. It follows that if $S_{1}$ and $S_{2}$ are any two of these sets, then $d\left(\left[x_{1}, \mathfrak{B}_{1}\right],\left[x_{2}, \mathfrak{B}_{2}\right]\right)$ has a value $d_{12}$ which is independent of the choice of $\left[x_{1}, \mathfrak{B}_{1}\right]$ in $S_{1}$ and $\left[x_{2}, \mathfrak{B}_{2}\right]$ in $S_{2}$. Set $d\left(S_{1}, S_{2}\right)=d_{12}$. Then $d\left(S_{1}, S_{2}\right)$ has all the properties of a distance in the class of sets $S$. Each of the sets $S$ is termed a continuous surface of the type of the circular disc. Any one 
of the continuous triples $[x(u), \mathfrak{B}]$ in $S$ is termed a (parametric) representation for the surface $S$. The distance $d\left(S_{1}, S_{2}\right)$ is known as the Fréchet distance of the surfaces $S_{1}$ and $S_{2}$. A sequence of surfaces $S_{n}$ is said to converge to the surface $S_{0}$ if $d\left(S_{n}, S_{0}\right)$ converges to zero. If $S_{n}$ converges to $S_{0}$, and if $\left[x_{0}(u)\right.$, $\left.\mathfrak{B}_{0}\right]$ be any representation for $S_{0}$, then there exist representations $\left[x_{n}(u), \mathfrak{B}_{n}\right]$ for $S_{n}$ such that $\mathfrak{B}_{n}$ is identical with $\mathfrak{B}_{0}$ for every $n$ and $x_{n}(u)$ converges on $\mathfrak{B}_{0}$ uniformly to $x_{0}(u)$.

2. Amongst the representations for a surface $S$, there may occur one $[x(u), \mathfrak{B}]$ of the form 1.

$$
x(u)=\left(u^{1}, u^{2}, x^{3}\left(u^{1}, u^{2}\right)\right), \quad u=\left(u^{1}, u^{2}\right) \text { in } \mathfrak{B} .
$$

Denote by ${ }^{3} \mathfrak{B}$ the image of $\mathfrak{B}$ in the ${ }^{3} x$-plane under the topological map $x^{1}=u^{2}, x^{2}=u^{1}$ for $\left(u^{1}, u^{2}\right)$ in $\mathfrak{B}$. Then

$$
x^{8}=x^{3}\left(x^{1}, x^{2}\right), \quad\left(x^{1}, x^{2}\right) \text { in }{ }^{3 \mathfrak{B}} .
$$

denotes what is commonly called a non-parametric representation for $S$. For this reason, the representation $[x(u), \mathfrak{B}]$ in 1 will be termed a representation of non-parametric origin for $S$. By symmetry, one should also term any representation for $S$ having one of the forms

$$
\begin{gathered}
\left(u^{2}, u^{1}, x^{3}\left(u^{1}, u^{2}\right)\right), \quad\left(u^{1}, x^{2}\left(u^{1}, u^{2}\right), u^{2}\right), \quad\left(u^{2}, x^{2}\left(u^{1}, u^{2}\right), u^{1}\right), \\
\left(x^{1}\left(u^{1}, u^{2}\right), u^{1}, u^{2}\right), \quad\left(x^{1}\left(u^{1}, u^{2}\right), u^{2}, u^{1}\right),
\end{gathered}
$$

a representation of non-parametric origin for $S$. But this is unnecessary, since any of these forms may be brought in to form 1 by a suitable change of notation. A surface need not have a representation of non-parametric origin, but if it does, that representation is unique.

3. Amongst the representations for a surface $S$, there may occur one $[x(u), \mathfrak{B}]$ of the form

$$
\text { 1. } \quad x(u)=\left(x^{1}\left(u^{1}, u^{2}\right), x^{2}\left(u^{1}, u^{2}\right), 0\right), \quad u=\left(u^{1}, u^{2}\right) \text { in } \mathfrak{B} \text {. }
$$

Geometrically speaking, such a surface lies entirely in the ${ }^{3} x$-plane. It is easily verified that any other representation for $S$ must have the form 1 . Such a surface is sometimes called a flat surface. The representation for $S$ defines a continuous transformation from the simple Jordan region $\mathfrak{B}$ in the $u$ plane to a bounded portion of the ${ }^{3} x$-plane.

4. Let $S$ be any continuous surface in $x$-space. If $[x(u), \mathfrak{B}]$ be any representation for $S$, then (see 4) $\left[{ }^{3} x(u), \mathfrak{B}\right]$ is a representation for a flat surface ${ }^{3} S$ in the ${ }^{3} x$-plane, which is the projection of $S$ on that plane. It follows at once (see $\mathrm{I}, 1)$ that if $[\bar{x}(u), \bar{B}]$ is any other representation for $S$ then $\left[{ }^{3} \bar{x}(u), \bar{B}\right]$ is another representation for ${ }^{3} S$-that is, the surface ${ }^{3} S$ is uniquely determined by $S$. Thus a continuous surface $S$ in $x$-space determines uniquely three flat projection surfaces ${ }^{1} S,{ }^{2} S,{ }^{3} S$ on the coordinate planes ${ }^{1} x,{ }^{2} x,{ }^{3} x$, respectively. 
5. A real, finite-valued function $f(u)=f\left(u^{1}, u^{2}\right)$ defined on a simple polygonal region $\mathfrak{B}$ in the $u$-plane is termed quasi-linear if $f(u)$ is continuous in $\mathfrak{B}$, and if there exists a triangulation of $\mathfrak{B}$ such that $f(u)$ is a linear function of $u^{1}$ and $u^{2}$ on each triangle of the triangulation. A continuous surface is termed a polyhedron, and denoted by $P$, if it possesses a representation $[x(u), \mathfrak{B}]$ such that $\mathfrak{B}$ is a simple polygonal region and each $x^{i}(u)$ is quasi-linear on $\mathfrak{B}$. Then there exists a triangulation of $\mathfrak{B}$ such that each of the functions $x^{i}(u)$ for $i=1,2,3$ is linear on every triangle in the triangulation. The image of each triangle in this triangulation is a (possibly degenerate) triangle; the sum of the areas of these image triangles is termed the elementary area of $P$-denote it by $a(P)$ Elementary considerations show that $a(P)$ depends only on the polyhedron $P$.

6. Let $S$ be any continuous surface. Then there always exist sequences of polyhedra $P_{n}$ such that $P_{n}$ converges to $S$; lim inf $a\left(P_{n}\right)$ is an upper bound for the Lebesgue area $A(S)$ of the surface; $A(S)$ is the greatest lower bound of all the upper bounds derived in this way. The Lebesgue area possesses the following important properties (see Rado [2, 3]).

1. If $P$ be any polyhedron, then $A(P)=a(P)$.

2. There exists a sequence of polyhedra $P_{n}$ such that $P_{n}$ converges to $S$ and $A\left(P_{n}\right)$ converges to $A(S)$.

3. The Lebesgue area $A(S)$ is a lower semi-continuous functional of $S$.

7. Much of the literature on continuous surfaces restricts its considerations to surfaces having representations of non-parametric origin (see $I, 2)$; in defining the "Lebesgue area" for such surfaces, it has been convenient to restrict the class of approximating polyhedra $P_{n}$ to have representations of non-parametric origin also. Let $A_{*}(S)$ denote the area of a surface $S$ having a representation of non-parametric origin when the class of approximating polyhedra is so restricted. Then clearly $A(S) \leqq A_{*}(S)$, and it is important in comparing the literature to know that the sign of equality always holds. This fact is implicit in the work of Rado (see Rado [1]), but no explicit proof seems to be in the literature. Such a proof will be a corollary to one of the results in this paper (see IV, 15).

8. Let $S$ be any continuous surface. The following principle has been advanced by Rado and Reichelderfer to direct their work in the theory of continuous surfaces (see $\mathrm{R}^{2}$ [2]). Assume that some sort of area-denote it by $A(S)$-is defined for $S$ and that, for each representation $[x(u), \mathfrak{B}]$ of $S$, some sort of Jacobians are defined for each of the projection representations $\left[{ }^{1} x, \mathfrak{B}\right],\left[{ }^{2} x, \mathfrak{B}\right],\left[{ }^{3} x, \mathfrak{B}\right]$ on the coordinate planes (see 4 )-denote these by ${ }^{1} \mathcal{F}(u),{ }^{2} \mathcal{F}(u),{ }^{3} \mathcal{F}(u)$, respectively, wherever they exist. Then a representation $[x(u), \mathfrak{B}]$ for $S$ is said to be absolutely continuous $(\mathcal{A}, \mathcal{F})$ provided that each of the Jacobians ${ }^{1} \mathcal{F}(u),{ }^{2} \mathcal{F}(u),{ }^{3} \mathcal{F}(u)$ exists almost everywhere in the interior $\mathfrak{B}^{0}$ of $\mathfrak{B}$, is summable on $\mathfrak{B}^{0}$, and 


$$
\mathcal{A}(S)=\int_{\mathfrak{O}_{0}}\left[{ }^{1} \mathcal{F}(u)^{2}+{ }^{2} \mathcal{F}(u)^{2}+{ }^{3} \mathcal{F}(u)^{2}\right]^{1 / 2} d u .
$$

\section{Chapter II}

\section{ON CONTINUOUS TRANSFORMATIONS IN THE PLANE}

In sections 1-9, 13, the salient features of the theory of bounded variation and absolute continuity for continuous transformations in the plane developed by Rado and Reichelderfer (see $\mathrm{R}^{2}$ [1]) are summarized. For all details, the reader is referred to the cited paper $\left({ }^{4}\right)$. Minor notational changes have been made to place the results in a form more convenient for the purposes of this paper. In sections 10-12, 14-22 extensions of this theory are discussed.

1. Let $\xi$ be any plane in $x$-space; on $\xi$ choose a rectangular coordinate system $\xi^{1}, \xi^{2}$, and adopt notations similar to those introduced in 4 for the $u$-plane. Let $\mathfrak{D}$ be any bounded domain in the $u$-plane. If $\xi(u)=\left(\xi^{1}\left(u^{1}, u^{2}\right)\right.$, $\left.\xi^{2}\left(u^{1}, u^{2}\right)\right)$ be a pair of real, single-valued functions defined, continuous, and bounded in $\mathfrak{D}$, then $[\xi(u), \mathfrak{D}]$ defines a bounded continuous transformation $T$, which associates with every point $u$ in $\mathfrak{D}$ a point $\xi=\xi(u)$ in a bounded portion of the $\xi$-plane. If $E$ be any set in the $u$-plane, let $T(E)$ denote the set of all points $\xi_{0}$ in the $\xi$-plane for which there exists a point $u_{0}$ in $E$ such that $\xi\left(u_{0}\right)=\xi_{0}$. If $\bar{E}$ be any set in the $\xi$-plane, let $T^{-1}(\bar{E})$ denote the set of all points $u_{0}$ in $\mathfrak{D}$ such that $\xi\left(u_{0}\right)$ is in $\bar{E}$. If $T_{1}:\left[\xi_{1}(u), \mathfrak{D}_{1}\right] ; T_{2}:\left[\xi_{2}(u), \mathfrak{D}_{2}\right]$ are two bounded continuous transformations, then their distance $\rho\left(T_{1}, T_{2} ; E\right)$ on any set $E$ in both $\mathfrak{D}_{1}$ and $\mathfrak{D}_{2}$ is the least upper bound of $\left\|\xi_{1}(u)-\xi_{2}(u)\right\|$ for $u$ in $E$. For any set $E$ in the $u$-plane, and for any point $\xi_{0}$ in the $\xi$-plane, $N\left(\xi_{0}, T, E\right)$ is defined to be the number (possibly $+\infty$ ) of points in the set $T^{-1}\left(\xi_{0}\right) \cdot E$. For fixed $\xi$ and $T, N(\xi, T, E)$ is a non-negative completely additive set function.

2. If $\Re$ be any Jordan region in $\mathfrak{D}$, and if $k$ be any non-negative integer, define $\bar{\Re}(k, T, \Re)$ to be the set of those points $\xi_{0}$ in the $\xi$-plane for which there exists a positive number $\epsilon$ such that $N\left(\xi_{0}, T_{*}, \Re\right) \geqq k$ for every bounded continuous transformation $T_{*}$ satisfying $\rho\left(T_{*}, T ; \Re\right)<\epsilon$. Clearly $\bar{\AA}(k, T, \Re)$ contains $\bar{\Re}(k+1, T, \Re)$ for $k=0,1,2, \ldots$ Define

$$
\bar{\Omega}(\infty, T, \Re)=\prod_{k=0}^{\infty} \bar{\Omega}(k, T, \Re) .
$$

A function $\mathrm{K}(\xi, T, \Re)$ is defined by the relations

$$
\mathbf{K}(\xi, T, \Re)=\left\{\begin{array}{rlll}
k & \text { on } & \bar{\Omega}(k, T, \Re)-\bar{\AA}(k+1, T, \Re) ; \\
+\infty & \text { on } & \bar{\Omega}(\infty, T, \Re) .
\end{array}\right.
$$

(4) The introduction of the $R^{2}$ paper contains a summary of their results, together with reference to the location of the proofs. 
Given a domain $\mathscr{D}$ in $\mathscr{D}$ let $\Re_{n}$ be a sequence of Jordan regions whose interiors fill up $\mathcal{D}$ from the interior (see 4 ). For fixed $\xi$ and $T$, the sequence $\mathrm{K}\left(\xi, T, \Re_{n}\right)$ has a limit (possibly $+\infty$ ) which is independent of the choice of the sequence of regions $\Re_{n}$ whose interiors fill up $\mathcal{D}$. This limit is denoted by $K(\xi, T, \mathcal{D})$, and is termed the essential multiplicity of $\xi$ under $T$ with respect to $\mathcal{D}$. It has the following properties.

1. The essential multiplicity $\mathrm{K}(\xi, T, \mathcal{D})$, for fixed $T$ and $\mathcal{D}$, is a lower semicontinuous function of $\xi$.

2. If $\mathcal{D}_{n}$ is a sequence of domains filling up $\mathcal{D}$ from the interior, if $T_{n}:\left[\xi_{n}(u)\right.$, $\left.\mathscr{D}_{n}\right]$ is a sequence of bounded continuous transformations such that for every closed set $F$ in $\mathcal{D}$ it is true that $\lim \rho\left(T_{n}, T ; F\right)=0$, then for every $\xi$ one has $\lim \inf \mathrm{K}\left(\xi, T_{n}, \mathcal{D}_{n}\right) \geqq \mathrm{K}(\xi, T, \mathcal{D})$. In particular, if $T_{n}$ is given by $\left[\xi(u), \mathcal{D}_{n}\right]$ then $\lim \mathrm{K}\left(\xi, T_{n}, \mathcal{D}_{n}\right)=\mathrm{K}(\xi, T, \mathcal{D})$.

3. For any Jordan region $\Re$ in $\mathfrak{D}$, it is true that $\mathrm{K}\left(\xi, T, \Re^{0}\right)=\mathrm{K}(\xi, T, \Re)$.

3. A set $B$ in the $u$-plane is termed a base set for the transformation $T$ if it is measurable, and for every closed oriented square $s$ whose interior $s^{0}$ is in $\mathfrak{D}$, the set $T\left(s^{0} \cdot \mathbb{B}\right)$ is measurable. Let $\mathbb{B}$ be any base set for $T$. Define, for any closed oriented square $s$ whose interior $s^{0}$ is in $\mathfrak{D}$

1.

$$
G(s, T, B)=\left|T\left(s^{0} \cdot \mathrm{B}\right)\right| \text {. }
$$

The transformation $T$ is said to be of bounded variation with respect to the base set $\mathbb{B}$ - briefly, BV $\mathbb{B}$-if there exists a finite positive constant $M$ such that for any finite system of nonoverlapping, closed, oriented squares $s_{i}$ whose interiors are in $\mathfrak{D}$, it is true that

$$
\sum G\left(s_{i}, T, \mathscr{B}\right)<M \text {. }
$$

The transformation $T$ is said to be absolutely continuous with respect to the base set $\mathbb{B}$-briefly, AC $\mathbb{B}$-if for every positive number $\epsilon$, there exists a positive number $\eta_{\epsilon}$ such that

$$
\sum G\left(s_{i}, T, B\right)<\epsilon
$$

for every finite system of nonoverlapping, closed, oriented squares $s_{i}$ whose interiors are in $\mathfrak{D}$ and for which

$$
\sum\left|s_{i}\right|<\eta_{e}
$$

If $T$ is $\mathrm{AC} B$, then it follows that $T$ is $\mathrm{BV} \mathcal{B}$.

Lemma. If $\mathcal{B}_{1}$ and $\mathscr{B}_{2}$ are base sets for $T$, then $\mathrm{B}=\mathbb{B}_{1}+\mathcal{B}_{2}$ is a base set for $T$. A necessary and sufficient condition that $T$ be $\mathrm{BV} B$ is that $T$ be both $\mathrm{BV} \mathrm{B}_{1}$ and $\mathrm{BV} \mathrm{B}_{2}$. A necessary and sufficient condition that $T$ be $\mathrm{AC} B$ is that $T$ be both $\mathrm{AC} \mathrm{B}_{1}$ and $\mathrm{AC} \mathrm{B}_{2}$.

4. A necessary and sufficient condition that a bounded continuous trans- 
formation $T$ be $\mathrm{BV} \mathscr{B}$ is that $N(\xi, T, \mathfrak{D} \cdot \mathbb{B})$ be summable $\left(^{(5)}\right.$. If $T$ is $\mathrm{BV} \mathscr{B}$, then $N(\xi, T, E \cdot \mathbb{B})$ is measurable and summable for any open or closed set $E$ relative to $\mathfrak{D}$. If $T$ is $\mathrm{BV} \mathscr{B}$, then the function of squares $G(s, T, \mathcal{B})$ defined in II, 3,1 possesses a derivative $D(u, T, \mathscr{B})$ almost everywhere in $\mathfrak{D}$. This derivative is summable in $\mathfrak{D}$, and one has, on every open set $O$ in $\mathfrak{D}\left(^{6}\right)$

$$
\int_{0} D(u, T, \mathcal{B}) d u \leqq \int N(\xi, T, O \cdot \mathcal{B}) d \xi .
$$

If $T$ is $\mathrm{AC} B$, then the sign of equality holds here. Conversely, if the sign of equality holds here for $O \equiv \mathfrak{D}$, then it holds for every open set $O$ in $\mathfrak{D}$, and $T$ is AC $\mathbb{B}$. If $T$ is $A C \mathscr{B}$, and if $E$ is any measurable set in $\mathfrak{D}$, then $N(\xi, T, E \cdot \mathbb{B})$ is measurable and summable; thus if $H(\xi)$ is a finite-valued, measurable function, then $H(\xi) N(\xi, T, E, \mathbb{B})$ is a measurable function. Under these conditions, it is also true that $H(\xi(u)) D(u, T, \mathscr{B})$ is measurable in $\mathfrak{D}$. Finally, if the transformation $T$ is $\mathrm{AC} \mathscr{B}$; the function $H(\xi)$ :is finite-valued and measurable; the set $E$ in $\mathfrak{D}$ is measurable; one of $H(\xi) N(\xi, T, E, \mathbb{B})$, $H(\xi(u)) D(u, T, \mathbb{B})$ is summable, then both of these functions are summable, and

1.

$$
\int_{E} H(\xi(u)) D(u, T, \mathcal{B}) d u=\int H(\xi) N(\xi, T, E \cdot \mathcal{B}) d \xi .
$$

5. Rado and Reichelderfer studied closely the notions of $B V \mathscr{B}$ and $A C \mathscr{B}$ for a certain choice of the base set $\mathbb{B}$ which is now described. Let $\Re$ be any Jordan region in $\mathfrak{D}$ (see 4 ). Let $\xi_{0}$ be any point in the $\xi$-plane not on the image under $T$ of the boundary of $\Re$. If $C$ be one of the curves bounding $\Re$, then the image of $C$ under $T$, taken as $u$ traverses $C$ in a positive sense relative to $\Re$, is a directed, closed continuous curve $\bar{C}$ not passing through $\xi_{0}$; consequently $\xi_{0}$ has a well defined topological index with respect to $\bar{C}$. The sum of these indices taken over all the boundary curves of $\Re$ is denoted by $\mu\left(\xi_{0}, T, \Re\right)$. For points $\xi_{*}$ on the image under $T$ of the boundary of $\Re$, one puts $\mu\left(\xi_{*}, T, \Re\right)=0$.

6 . Let $\xi_{0}$ be any point in the $\xi$-plane. The set $T^{-1}\left(\xi_{0}\right)$ is a closed set relative to $\mathfrak{D}$, hence decomposes in a unique way into components which are maximal connected closed sets relative to $\mathfrak{D}$. If a component of $T^{-1}\left(\xi_{0}\right)$ has a positive distance from the boundary of $\mathfrak{D}$, then it is a connected closed set in the absolute sense-that is, a continuum; such a component is termed a maximal model continuum for $\xi_{0}$ under $T$ in $\mathfrak{D}$, and is denoted generically by $\sigma\left(\xi_{0}, T\right)$. A $\sigma\left(\xi_{0}, T\right)$ is termed essential if in every open neighborhood of $\sigma\left(\xi_{0}, T\right)$, there is a Jordan region $\Re$ containing $\sigma\left(\xi_{0}, T\right)$ in its interior and for

(5) Since all functions considered in the $\xi$-plane are zero outside a sufficiently large disc, they are termed summable whenever they are summable on such a disc, and no range of integration will be explicitly indicated.

(6) See Footnote 5. 
which $\mu\left(\xi_{0}, T, \Re\right)$ is not zero. If $\mathcal{D}$ be any subdomain in $\mathfrak{D}$, then the number of essential maximal model continua $\sigma\left(\xi_{0}, T\right)$ for $\xi_{0}$ under $T$ in $\mathcal{D}$ is equal to the essential multiplicity $\mathrm{K}\left(\xi_{0}, T, \mathcal{D}\right)$ (see II, 2). If $\Re$ be any Jordan region in $\mathfrak{D}$ for which $\mu\left(\xi_{0}, T, \Re\right)$ is not zero, then $\xi_{0}$ has an essential maximal model continuum under $T$ in the interior of $\Re$.

7. If $\mathcal{D}$ be any domain in $\mathfrak{D}$, then denote by $E(T, \mathcal{D})$ the set of all points $u_{0}$ which belongs to some essential maximal model continuum for $\xi\left(u_{0}\right)$ under $T$ in $\mathcal{D}$. Denote by $\mathcal{E}(T, \mathcal{D})$ that subset of $E(T, \mathcal{D})$ which consists of all those points $u_{0}$ which themselves constitute essential maximal model continua for $\xi\left(u_{0}\right)$ under $T$ in $\mathcal{D}$; evidently $\mathcal{E}(T, \mathcal{D})=\mathcal{E}(T, \mathfrak{D}) \cdot \mathcal{D}$, but a similar formula does not generally hold for $E(T, \mathcal{D})$. If $u_{0}$ be any point of $\mathcal{E}(T, \mathfrak{D})$ which has a neighborhood free of points belonging to other essential maximal model continua for $\xi\left(u_{0}\right)$ under $T$ in $\mathfrak{D}$, then it is true that $\mu\left(\xi\left(u_{0}\right), T, \Re\right)$ has a nonzero value independent of the choice of a Jordan region $\Re$ in this neighborhood which contains, $u_{0}$ in its interior and whose boundary contains no point of $T^{-1}\left(\xi\left(u_{0}\right)\right)$; denote this value by $j\left(u_{0}, T\right)$. For all points $u_{*}$ in $\mathfrak{D}$ not having the properties of $u_{0}$, set $j\left(u_{*}, T\right)$ equal to zero. Then $j(u, T)$ is a Baire function in $\mathfrak{D}$ and $|j(u, T)|$ does not exceed one, except possibly on a denumerable set of points in $\mathfrak{D}$.

8. It is the set $\mathcal{E}(T, \mathfrak{D})$ which Rado and Reichelderfer employ for a base set (see II, 3 ); it is the set $E(T, \mathfrak{D})$ which plays a prominent role in the following theory. Because the results for these two base sets are so closely related, the results for the set $\mathcal{E}(T, D)$ developed by Rado and Reichelderfer are now summarized as a basis for stating and proving results for the set $E(T, \mathfrak{D})$. Let $K_{0}$ denote the class of all bounded continuous transformations $T:[\xi(u)$, $\mathfrak{D}]$ which are BV $\mathcal{E}(T, \mathfrak{D})$. Let $K_{1}$ denote that subclass of $K_{0}$ (see II, 3) consisting of all transformations $T$ which are $\mathrm{AC} \mathcal{E}(T, \mathfrak{D})$. Let $K_{2}$ denote the class of all transformations $T$ in $K_{1}$ for which the relation $N(\xi, T, \mathcal{E}(T, \mathfrak{D}))$ $=\mathrm{K}(\xi, T, \mathfrak{D})$ holds almost everywhere. Finally, let $K_{3}$ denote the class of all transformations $T$ in $K_{2}$ for which the ordinary Jacobian

$$
J(u, T)=\partial\left(\xi^{1}, \xi^{2}\right) / \partial\left(u^{1}, u^{2}\right), \quad u=\left(u^{1}, u^{2}\right) \text { in } \mathfrak{D},
$$

exists almost everywhere in $\mathfrak{D}$. If $T$ is in $K_{0}$, then $D(u, T, \mathcal{E}(T, \mathfrak{D}))$ exists almost everywhere in $\mathfrak{D}$ and is summable on $\mathfrak{D}$ (see II, 3 ) - denote this derivative by $D(u, T)$. Define

$$
\mathcal{F}(u, T)=\left\{\begin{array}{cl}
j(u, T) D(u, T) & \text { wherever } D(u, T) \text { exists; } \\
0 & \text { otherwise. }
\end{array}\right.
$$

The function $\mathcal{F}(u, T)$ is termed the generalized Jacobian for the transformation $T$. From II, 7 it follows that $|\mathcal{F}(u, T)| \leqq D(u, T)$ almost everywhere in $\mathfrak{D}$, and $\mathcal{F}(u, T)$ is measurable in $\mathfrak{D}$; hence $\mathcal{F}(u, T)$ is summable in $\mathfrak{D}$. If $T$ is in the class $K_{2}$, it follows that $|\mathcal{F}(u, T)|=D(u, T)$ almost everywhere in $\mathfrak{D}$. If $T$ is in the class $K_{3}$, it is true that $\mathcal{F}(u, T)=J(u, T)$ almost everywhere in $\mathfrak{D}$. 
The class $K_{3}$ contains all bounded continuous transformations $T:[\xi(u), \mathfrak{D}]$ which satisfy a Lipschitz condition in the following restricted sense: there exists a finite constant $L$ such that if $u_{1}$ and $u_{2}$ are any two points of $\mathfrak{D}$ for which the line segment joining them is contained in $\mathfrak{D}$, then $\left\|\xi\left(u_{1}\right)-\xi\left(u_{2}\right)\right\|$ $\leqq L\left\|u_{1}-u_{2}\right\|$. If $T$ is a bounded continuous transformation for which the ordinary Jacobian exists almost everywhere in $\mathfrak{D}$, and for which $\mathrm{K}(\xi, T, \mathfrak{D})$ is summable, then $D(u, T) \geqq|J(u, T)|$ almost everywhere in $\mathfrak{D}\left({ }^{7}\right)$.

9. Combining results stated in II, 4, 8, one obtains the

1. Leмma. Let $T:[\xi(u), \mathfrak{D}]$ be a bounded continuous transformation for which $\mathrm{K}(\xi, T, \mathfrak{D})$ is summable. Then $\mathcal{F}(u, T)$ exists almost everywhere in $\mathfrak{D}$, is summable on $\mathfrak{D}$, and

$$
\int \mathrm{K}(\xi, T, \mathcal{D}) d \xi \geqq \int N(\xi, T, \mathcal{E}(T, \mathcal{D})) d \xi \geqq \int_{\mathcal{D}} D(u, T) d u \geqq \int_{\mathcal{D}}|\mathcal{F}(u, T)| d u
$$

for every domain $\mathcal{D}$ in $\mathfrak{D}$. A necessary and sufficient condition that all the signs of equality hold here for $\mathcal{D} \equiv \mathfrak{D}$ is that $T$ be in the class $K_{2}$. When $T$ is in $K_{2}$, all the signs of equality also hold for every domain $D$ in $\mathfrak{D}$.

2. LеммA. Let $T:[\xi(u), \mathfrak{D}]$ be a bounded continuous transformation for which $\mathrm{K}(\xi, T, \mathfrak{D})$ is summable, and for which the ordinary Jacobian $J(u, T)$ exists almost everywhere in $\mathfrak{D}$. Then $J(u, T)$ is summable on $\mathfrak{D}$, and

$$
\int \mathrm{K}(\xi, T, \mathcal{D}) d \xi \geqq \int N(\xi, T, \mathcal{E}(T, \mathcal{D})) d \xi \geqq \int_{\mathcal{D}} D(u, T) d u \geqq \int_{\mathcal{D}}|J(u, T)| d u
$$

for every domain $\mathcal{D}$ in $\mathfrak{D}$. A necessary and sufficient condition that all the signs of equality hold here for $\mathcal{D} \equiv \mathfrak{D}$ is that $T$ be in the class $K_{3}$, When $T$ is in $K_{3}$, all the signs of equality also hold for every domain $\mathcal{D}$ in $\mathfrak{D}$.

10. Lemma. If $T:[\xi(u), \mathfrak{D}]$ be any bounded continuous transformation, then the set $E(T, \mathfrak{D})($ see II, 7) is a product of open sets, hence a Borel set.

Proof. Let $n$ be any positive integer. Denote by $E_{n}$ the set of points $u_{0}$ in $\mathfrak{D}$ for each of which there exists a Jordan region $\Re$ in $\mathfrak{D}$ satisfying the following conditions: $u_{0}$ lies in the interior of $\Re ; T(\Re)$ lies in the open disc $\left\|\xi-\xi\left(u_{0}\right)\right\|<n^{-1} ; \mu\left(\xi\left(u_{0}\right), T, \Re\right) \neq 0$. Evidently each $E_{n}$ is an open set. One easily verifies that $\prod E_{n}=E(T, \mathfrak{D})$, so the lemma is established.

Set $e(T, \mathfrak{D})=E(T, \mathfrak{D})-\varepsilon(T, \mathfrak{D})$ - that is, $e(T, \mathfrak{D})$ is the set of those points $u_{0}$ belonging to some nondegenerate essential maximal model continuum for $\xi\left(u_{0}\right)$ under $T$ in $\mathfrak{D}$. Rado and Reichelderfer have shown that $\mathcal{E}(T, \mathfrak{D})$ is also a product of open sets, hence a Borel set. Thus $e(T, D)$ is a Borel set. By a general theorem (see Kuratowski [1, p. 249]) it follows that for every choice of an open square $s^{0}$ in the $u$-plane, the sets $T\left(s^{0} \cdot e(T, \mathfrak{D})\right), T\left(s^{0} \cdot E(T, \mathfrak{D})\right)$ are

( 7 ) The last result is established in $\$ 5.6$ of the $R^{2}$ paper. 
both measurable. Thus both the sets $e(T, \mathfrak{D}), E(T, \mathfrak{D})$ may serve as base sets (see II, 3), and the general theory in II, 3, 4 is applicable.

11. Lemma. A necessary and sufficient condition that the set $T(e(T, \mathfrak{D}))$ be of measure zero is that $T$ be $\mathrm{BV} e(T, \mathfrak{D})$. Whenever $T$ is $\mathrm{BV} e(T, \mathfrak{D}))$, it is also $\mathrm{AC} e(T, \mathfrak{D})$.

Proof. Observe that (see II, 1)

$$
N(\xi, T, e(T, \mathfrak{D}))=\left\{\begin{array}{cl}
+\infty & \text { for } \xi \text { in } T(e(T, \mathfrak{D})) \\
0 & \text { otherwise. }
\end{array}\right.
$$

Thus a necessary and sufficient condition that $N(\xi, T, e(T, \mathfrak{D}))$ be summable is that $|T(e(T, \mathfrak{D}))|=0$; in view of the facts in II, 4, this establishes the first part of the lemma. If $T$ is $\mathrm{BV} e(T, \mathfrak{D}))$, it follows at once that

$$
0 \leqq \int_{\mathscr{D}} D(u, T, e(T, \mathfrak{D})) d u \leqq \int N(\xi, T, e(T, \mathfrak{D})) d \xi=0,
$$

so that the sign of equality holds here, and $T$ is $\mathrm{AC} e(T, \mathfrak{D})$.

From this lemma and the lemma in II, 3 follows the

CoROllary. Let $T$ be any bounded continuous transformation which is $\mathrm{BV} e(T, \mathfrak{D}) . A$ necessary and sufficient condition that $T$ be $\mathrm{BV} E(T, \mathfrak{D})$ is that $T$ be $\mathrm{BV} \mathcal{E}(T, \mathfrak{D})$. A necessary and sufficient condition that $T$ be $\mathrm{AC} E(T, \mathfrak{D})$ is that $T$ be $\operatorname{AC} \mathcal{E}(T, D)$.

If $T$ is $\mathrm{BV} e(T, \mathfrak{D})$ it is evident that (see II, 3, 7, 10) for every closed oriented square $s$ whose interior is in $\mathfrak{D}$,

$$
G(s, T, E(T, \mathfrak{D}))=G(s, T, \mathcal{E}(T, \mathfrak{D})) .
$$

Thus whenever $T$ is $\mathrm{BV} e(T, \mathfrak{D})$, it follows that $D(u, T, E(T, \mathfrak{D}))=D(u, T$, $\mathcal{E}(T, \mathfrak{D})) \equiv D(u, T)$ almost everywhere in $\mathfrak{D}$ (see II, 4, 8).

12. Let $T:[\xi(u), D]$ be a bounded continuous transformation. From the definitions in II, 1, 2,6, 7, it is clear that

1.

$$
N(\xi, T, E(T, \mathfrak{D})) \geqq \mathrm{K}(\xi, T, \mathfrak{D}) \geqq N(\xi ; T, \mathcal{E}(T, \mathfrak{D})),
$$

and the sign of inequality holds between any two of these three functions if and only if $\xi$ lies in the set $T(e(T, D))$ and one of the functions involved is finite. Since $\mathrm{K}(\xi, T, \mathfrak{D})$ is measurable (see II, 2,1$)$, it follows that a necessary condition that $\mathrm{K}(\xi, T, \mathfrak{D})$ be summable is that $T$ be BV $\mathcal{E}(T, \mathfrak{D})$, while a sufficient condition that $\mathrm{K}(\xi, T, \mathfrak{D})$ be summable is that $T$ be $\mathrm{BV} E(T, \mathfrak{D})$; in this latter case, the signs of equality in relation 1 hold almost everywhere. Now suppose that $T$ is $\mathrm{BV} \mathcal{E}(T, \mathfrak{D})$; then since $N(\xi, T, \mathcal{E}(T, \mathfrak{D}))$ is finite almost everywhere, one concludes that a necessary and sufficient condition that $\mathrm{K}(\xi, T, \mathfrak{D})=N(\xi, T, \mathcal{E}(T, \mathfrak{D}))$ almost everywhere is that $T$ be $\operatorname{BV} E(T, \mathfrak{D})$. 
In view of the definition of the class $K_{2}$ (see II, 8), the corollary in the preceding section, and these remarks, one obtains the

LEMMA. $A$ necessary and sufficient condition that a bounded continuous transformation $T:[\xi(u), \mathfrak{D}]$ be in the class $K_{2}$ is that $T$ be $\mathrm{AC} E(T, \mathfrak{D})$.

13. The lemma in the preceding section gives a simple characterization of transformations in the class $K_{2}$ and makes available all the results established by Rado and Reichelderfer for this class whenever $T$ is $\operatorname{AC~} E(T, \mathfrak{D})$. For example, they have the

Closure theorem. Let there be given bounded domains $\mathcal{D}$ and $\mathcal{D}_{n}$ in the $u$-plane and bounded continuous transformations $T:[\xi(u), \mathcal{D}]$ and $T_{n}:\left[\xi_{n}(u)\right.$, $\left.\mathcal{D}_{n}\right], n=1,2, \cdots$, with the following properties: (i) the domains $\mathcal{D}_{n}$ fill up $\mathcal{D}$ from the interior (see 4); (ii) the generalized Jacobian $\mathcal{F}(u, T)$ exists almost everywhere in $\mathcal{D}$ and is summable on $\mathcal{D}$ (see II, 8); (iii) $T_{n}$ is in $K_{2}$ for $n=1$, $2, \cdots$; (iv) for every closed set $F$ contained in $\mathcal{D}$, it is true that

$$
\lim \rho\left(T_{n}, T ; F\right)=0, \quad \lim \int_{F}\left|\mathcal{F}\left(u, T_{n}\right)-\mathcal{F}(u, T)\right| d u=0 .
$$

Then $T$ is in $K_{2}$.

Using the preceding results, this theorem may be restated and improved as follows.

14. MODIFIED CLOSURE THEOREM. Let there be given bounded domains $\mathcal{D}$ and $\mathscr{D}_{n}$ in the $u$-plane and bounded continuous transformations $T:[\xi(u), \mathcal{D}]$ and $T_{n}:\left[\xi_{n}(u), \Phi_{n}\right], n=1,2, \cdots$, with the following properties: (i) the domains $\mathscr{D}_{n}$ fill up $\mathcal{D}$ from the interior; (ii) the generalized Jacobian $\mathcal{F}(u, T)$ exists almost everywhere in $\mathcal{D}$ and is summable on $D$; (iii) $T_{n}$ is AC $E\left(T_{n}, \mathscr{D}_{n}\right)$ for $n=1,2, \ldots ;$ (iv $\left.^{\prime}\right)$ there exists a sequence of Jordan regions $\Re_{m}$ in $\Phi$ whose interiors $\Re_{m}^{0}$ fill up $\mathcal{D}$ from the interior, and such that

$$
\begin{array}{r}
\lim _{n \rightarrow \infty} \rho\left(T_{n}, T ; \Re_{m}\right)=0, \quad \lim _{n \rightarrow \infty} \int_{\Re_{m}}\left|\mathcal{F}\left(u, T_{n}\right)\right| d u=\int_{\Re_{m}}|\mathcal{F}(u, T)| d u, \\
m=1,2, \cdots .
\end{array}
$$

Then $T$ is AC $E(T, \mathcal{D})$.

Inspection of the proof of Rado and Reichelderfer for their closure theorem reveals that property (iv'), which is a consequence of (iv), is all that is needed for that proof.

Corollary. Condition (iv') of the above theorem may be replaced by the following condition: (iv') for every closed oriented square $s$ contained in $\mathcal{D}$, it is true that 


$$
\lim \rho\left(T_{n}, T ; s\right)=0, \quad \lim \int_{\varepsilon}\left|\mathcal{F}\left(u, T_{n}\right)\right| d u=\int_{s}|\mathcal{F}(u, T)| d u
$$

Proof. Let $R$ denote any Jordan region in $\mathcal{D}$ which may be expressed as a sum of a finite number of nonoverlapping closed oriented squares $s$. From $\left(i v^{\prime \prime}\right)$ it follows that

$$
\lim \rho\left(T_{n}, T ; R\right)=0, \quad \lim \int_{R}\left|\mathcal{F}\left(u, T_{n}\right)\right| d u=\int_{R}|\mathcal{F}(u, T)| d u .
$$

By a known theorem in topology (see Kerékjártó [1]), there exists a sequence of regions $R_{n}$ whose interiors fill up $\mathcal{D}$ from the interior. Thus condition (iv") implies (iv'), and the corollary is established.

15. In view of the lemma in II, 12, the class $K_{3}$ defined in II, 8 may be characterized as the class of all transformations $T:[\xi(u), \mathfrak{D}]$ which are AC $E(T, D)$ and for which the ordinary Jacobian $J(u, T)$ exists almost everywhere in $\mathfrak{D}$. For the class $K_{3}$ another closure theorem is given by Rado and Reichelderfer, the proof of which is based upon the closure theorem for the class $K_{2}$ stated in II, 13. By using the modified closure theorem just given, one may parallel their proof to establish the

Modified CLOSURE THEOREM. Let there be given bounded domains $\mathscr{D}$ and $\mathscr{D}_{n}$ and bounded continuous transformations $T:[\xi(u), \mathcal{D}]$ and $T_{n}:\left[\xi_{n}(u), \mathcal{D}_{n}\right]$, $n=1,2, \cdots$, , with the following properties: (i) the domains $\mathcal{D}_{n}$ fill $u p \mathscr{D}$ from the interior; (ii) the ordinary Jacobian $J(u, T)$ exists almost everywhere in $D$ and is summable on $D_{\text {; }}$ (iii) $T_{n}$ is $\mathrm{AC} E\left(T_{n}, \mathscr{D}_{n}\right)$ and the ordinary Jacobians $J\left(u, T_{n}\right)$ exist almost everywhere in $\mathcal{D}_{n}$ for $n=1,2, \cdots$; (iv') there exists a sequence of Jordan regions $\Re_{m}$ in $\mathcal{D}$ whose interiors $\Re_{m}^{0}$ fill up $\mathcal{D}$ from the interior, and such that

$$
\begin{array}{r}
\lim _{n \rightarrow \infty} \rho\left(T_{n}, T ; \Re_{m}\right)=0, \quad \lim _{n \rightarrow \infty} \int_{\Re_{m}}\left|J\left(u, T_{n}\right)\right| d u=\int_{\Re_{m}}|J(u, T)| d u, \\
m=1,2, \ldots .
\end{array}
$$

Then $T$ is AC $E(T, \mathcal{D})$.

Corollary. Condition (iv') of the above theorem may be replaced by the following condition: (iv') for every closed oriented square sontained in $\mathcal{D}$, it is true that

$$
\lim \rho\left(T_{n}, T ; s\right)=0, \quad \lim \int_{2}\left|J\left(u, T_{n}\right)\right| d u=\int_{2}|J(u, T)| d u .
$$

If in these results, condition (ii) be weakened by dropping the requirement that the ordinary Jacobians $J\left(u, T_{n}\right)$ exist almost everywhere in $\mathcal{D}_{n}$ for $n=1,2, \cdots$, and if these Jacobians be replaced by the generalized Jacobians $\mathcal{F}\left(u, T_{n}\right)$ for $n=1,2, \cdots$, then the conclusions remain the same. 
16. The results to be established in the following sections are necessary for a comparison of certain notions in this paper with those in the literature. In the theory of bounded continuous transformations just sketched, the range of definition has been a bounded domain $\mathfrak{D}$ (see $I, 1$ ). In applications, one may have a continuous transformation given by $[\xi(u), \mathfrak{B}]$, where $\mathfrak{B}$ is a simple Jordan region (see 4 ) and $\xi(u)$ is a pair of functions defined and continuous on the closed set $\mathfrak{B}$. Evidently the transformation given by $\left[\xi(u), \mathfrak{B}^{0}\right]$ is then bounded and continuous. From work of Rado and Reichelderfer (see II, 2, 6), it follows that the transformations $[\xi(u), \mathfrak{B}]$ and $\left[\xi(u), \mathfrak{B}^{0}\right]$ have the same essential multiplicity functions and the same essential maximal model continua. Since the essential multiplicity and the essential maximal model continua play the basic role in this paper-that is, since the transformations behave essentially alike-there will be no confusion if one designates either of them by.T. In the sequel this is done, but it is to be understood that whenever preceding theory is applied, $T$ is to be interpreted as the transformation $\left[\xi(u), \mathfrak{B}^{0}\right]$.

17. LEMMA. Let $T:[\xi(u), \mathfrak{D}]$ be a bounded continuous transformation which is $\mathrm{BV} \mathscr{B}$, where $\mathcal{B}$ is an arbitrary base set (see II, 3). Given a positive number $\epsilon$, it is true that the number of mutually exclusive sets $E$, each of which is either an open set or a closed set relative to $\mathfrak{D}$, for which the measure of $T(E \cdot \mathcal{B})$ exceeds $\epsilon$ is finite.

Proof. Let $E_{1}, \cdots, E_{m}$ be any finite system of mutually exclusive sets having the required properties. Since $T$ is $\mathrm{BV} \mathscr{B}$, it follows that $N(\xi, T, \mathfrak{D} \cdot \mathbb{B})$, $N\left(\xi, T, E_{i} \cdot \mathbb{B}\right)$ are measurable and summable (see II, 4). The lemma follows from the inequalities

$$
\epsilon^{-1} \int N(\xi, T, \mathfrak{D} \cdot \mathbb{B}) d \xi \geqq \epsilon^{-1} \sum_{i=1}^{m} \int N\left(\xi, T, E_{i} \cdot \mathbb{B}\right) d \xi \geqq \epsilon^{-1} \sum_{i=1}^{m}\left|T\left(E_{i} \cdot \mathcal{B}\right)\right|>m .
$$

From this lemma comes the

CoROLlaRy. Let $T:[\xi(u), \mathfrak{D}]$ be a bounded continuous transformation which is BV B. Then the number of lines l in any family of parallel lines in the u-plane for which $T(l \cdot B)$ has positive measure is at most enumerable.

18. Let $T:[\xi(u), \mathfrak{B}]$ where $\mathfrak{B}$ is a simple Jordan region in the $u$-plane, be a continuous transformation. Let $\overline{\mathfrak{B}}$ be any other simple Jordan region in the $u$-plane, and consider any topological map of $\overline{\mathfrak{B}}$ on to $\mathfrak{B}$ given by $[\bar{u}(u), \overline{\mathfrak{B}}]$. Denote by $\bar{T}$ the continuous transformation $[\bar{\xi}(u), \overline{\mathfrak{B}}]$ where $\bar{\xi}(u)=\xi(\bar{u}(u))$ for $u$ in $\overline{\mathfrak{B}}$. Since the sets $E\left(T, \mathfrak{B}^{0}\right)$ and $E\left(\bar{T}, \overline{\mathfrak{B}}^{0}\right)$ are in biunique correspondence under the map, it is true that $N\left(\xi, T, E\left(T, \mathfrak{B}^{0}\right)\right)=N\left(\xi, \bar{T}, E\left(\bar{T}, \overline{\mathfrak{B}}^{0}\right)\right)$. This implies (see II, 4) the

Lemma. If $T$ is $\mathrm{BV} E\left(T, \mathfrak{B}^{0}\right)$, then $\bar{T}$ is $\mathrm{BV} E\left(\bar{T}, \overline{\mathfrak{B}}^{0}\right)$. 
19. Now let $T_{0}[\xi(u), \mathfrak{B}]$, where $\mathfrak{B}$ is a simple Jordan region in the $u$-plane, be a continuous transformation for which $\mathrm{K}\left(\xi, T, \mathfrak{B}^{0}\right)$ is summable (see II, 2 ). If $S(\mathfrak{B})$ be any finite system (see 4), denote by $C$ the set of points in $\mathfrak{B}^{0}$ but not in the interior of any $B$ belonging to $S(\mathfrak{B})$. Then (see II, 6), wherever $\mathrm{K}\left(\xi, T, \mathfrak{B}^{0}\right)$ is finite-hence almost everywhere,

1. $\mathrm{K}\left(\xi, T, \mathfrak{B}^{0}\right)\left\{\begin{array}{l}=\sum \mathrm{K}\left(\xi, T, B^{0}\right) \text { for } B \text { in } S(\mathfrak{B}) \text { if } \xi \text { is not in } T\left(C \cdot E\left(T, \mathfrak{B}^{0}\right)\right) ; \\ >\sum \mathrm{K}\left(\xi, T, B^{0}\right) \text { for } B \text { in } S(\mathfrak{B}) \text { if } \xi \text { is in } T\left(C \cdot E\left(T, \mathfrak{B}^{0}\right)\right) .\end{array}\right.$ Thus

2.

$$
\int \mathrm{K}\left(\xi, T, \mathfrak{B}^{0}\right) d \xi \geqq \sum \int \mathrm{K}\left(\xi, T, B^{0}\right) \text { for } \quad B \text { in } S(\mathfrak{B}),
$$

and a necessary and sufficient condition that the sign of equality hold here is that the sign of equality hold almost everywhere in relation 1 - that is, that the set $T\left(C \cdot E\left(T, \mathfrak{B}^{0}\right)\right)$ be of measure zero. For brevity, any finite system $S(B)$ for which the sign of equality holds in relation 2 is termed a maximal system for $T$.

20. Lemma. Given a continuous transformation $T:[\xi(u), \mathfrak{B}]$. A necessary and sufficient condition that, for any positive number $\delta$ there exist maximal systems $S(\mathfrak{B})$ for $T$ such that $\|S(\mathfrak{B})\|$ is less than $\delta$ is that $T$ be $\mathrm{BV} E\left(T, \mathfrak{B}^{0}\right)$.

Proof. First, assume that there exists a sequence of finite systems $S_{n}(\mathfrak{B})$ such that $\left\|S_{n}(\mathfrak{B})\right\|$ converges to zero and

$$
\int \mathrm{K}\left(\xi, T, \mathfrak{B}^{0}\right) d \xi=\sum \int \mathrm{K}\left(\xi, T, B^{0}\right) d \xi \quad \text { for } \quad B \text { in } S_{n}(\mathfrak{B}), \quad n=1,2, \cdots
$$

Denote $C_{n}$ the set of points in $\mathfrak{B}^{0}$ none of which is in the interior of a region $B$ belonging to $S_{n}(\mathfrak{B})$ for $n=1,2, \cdots$; set $\Gamma=\sum C_{n}$. From the remark in II, 19 , it follows that the set $T\left(\Gamma \cdot E\left(T, \mathfrak{B}^{0}\right)\right)$ is of measure zero. On the other hand, the set $T\left(e\left(T, \mathfrak{B}^{0}\right)\right.$ ) (see II, 10) is clearly a subset of $T\left(\Gamma \cdot E\left(T, \mathfrak{B}^{0}\right)\right.$ ), hence also of measure zero. By the lemma in II, 11, it is true that $T$ is $\mathrm{BV} e\left(T, \mathfrak{B}^{0}\right)$. Since $\mathrm{K}\left(\xi, T, \mathfrak{B}^{0}\right)$ is summable, it follows from II, 12 that $T$ is $\mathrm{BV} \mathcal{E}\left(T, \mathfrak{B}^{0}\right)$. From the lemma in $\mathrm{II}, 3$, it is clear that $T$ is $\mathrm{BV} E\left(T, \mathfrak{B}^{0}\right)$. This establishes the necessity of the condition. Next, assume that $T$ is BV $E\left(T, \mathfrak{B}^{0}\right)$; then $\mathrm{K}\left(\xi, T, \mathfrak{B}^{0}\right)$ is summable (see II, 12). First, consider the special case when the simple Jordan region $\mathfrak{B}$ is an interval $\Im$ (see 4 ). Given a positive number $\delta$, there exists, according to the corollary in II, 17, an interval subdivision $S(\Im)$ such that $\|S(\Im)\|$ is less than $\delta$ and $T\left(l \cdot E\left(T, \Im^{0}\right)\right.$ ) is of measure zero for every line $l$ forming the subdivision. Denote by $C$ the set of points in $\Im^{0}$ belonging to the lines of subdivision forming $S(\Im)$. Clearly $T\left(C \cdot E\left(T, \Im^{0}\right)\right.$ ) is of measure zero, so that $S(\Im)$ is a maximal system for $T$ with $\|S(\Im)\|$ less than $\delta$. Now consider the general case when 
$\mathfrak{B}$ is any simple Jordan region. Let $\Im$ be any interval in the $u$-plane; then there exists a topological map of $\Im$ onto $\mathfrak{B}$ given by $[\bar{u}(u), \Im]$. Denote by $\bar{T}$ the transformation $[\bar{\xi}(u), \Im]$ where $\bar{\xi}(u)=\xi(\bar{u}(u))$ for $u$ in $\Im$. Since $\bar{T}$ is BV $E\left(\bar{T}, \Im^{0}\right)$ (see II, 18), it follows by the result just established that there exist interval subdivisions $\bar{S}(\Im)$ which are maximal with respect to $\bar{T}$ for which $\|\bar{S}(\Im)\|$ is arbitrarily small. A maximal system $\bar{S}(\Im)$ for $\bar{T}$ corresponds under the map to a system $S(\mathfrak{B})$ which is easily seen to be maximal for $T$. In view of the uniform continuity of the topological map, $\|S(\mathfrak{B})\|$ will be less than $\delta$ provided $\|\bar{S}(\Im)\|$ is chosen sufficiently small. This establishes the lemma.

21. The methods of proof for the preceding lemma yield the

LEMma. If $T_{n}:\left[\xi_{n}(u), \mathfrak{B}\right], n=1,2, \cdots$, be a sequence of continuous transformations each defined on the simple Jordan region $\mathfrak{B}$, and each $\mathrm{BV} E\left(T_{n}, \mathfrak{B}\right)$, then for every positive number $\delta$ there exists a subdivision $S(\mathfrak{B})$ having $\|S(\mathfrak{B})\|$ less than $\delta$, such that $S(\mathfrak{B})$ is maximal for each of the $T_{n}$ for $n=1,2, \cdots$. If $\mathfrak{B}$ is an interval then $S(\mathfrak{B})$ may be chosen to be an interval subdivision.

22. Again, let $T:[\xi(u), \mathfrak{B}]$ be a continuous transformation. For any simple Jordan region $B$ in $\mathfrak{B}$, denote by $c\left(\Im, T, B^{0}\right)$ the characteristic function of the set of points $\xi$ where $\mathrm{K}\left(\xi, T, B^{0}\right)$ is positive (see II, 2). Then $c\left(\xi, T, B^{0}\right)$ is summable, and

$$
\int c\left(\xi, T, B^{0}\right) d \xi=\left|T\left(E\left(T, B^{0}\right)\right)\right|=|\Re(1, T, B)| \quad \text { for } B \text { in } \mathfrak{B} .
$$

Consider any sequence of finite systems $S_{n}(\mathfrak{B})$ for which $\left\|S_{n}(\mathfrak{B})\right\|$ converges to zero. Clearly

$$
\sum_{B \text { in } S_{n}(\mathfrak{B})}\left[\mathrm{K}\left(\xi, T, B^{0}\right)-c\left(\xi, T, B^{0}\right)\right]\left\{\begin{array}{l}
\leqq \mathrm{K}\left(\xi, T, \mathfrak{B}^{0}\right) ; \\
\rightarrow_{n \rightarrow \infty} 0 \text { if } \mathrm{K}\left(\xi, T, \mathfrak{B}^{0}\right) \text { is finite. }
\end{array}\right.
$$

Thus if $\mathrm{K}\left(\xi, T, \mathfrak{B}^{0}\right)$ is summable, it follows by a theorem of Lebesgue that one may integrate this sequence termwise to obtain

$$
\lim _{n \rightarrow \infty} \sum_{B \text { in } S_{n}(\xi)} \int\left[\mathrm{K}\left(\xi, T, B^{0}\right)-c\left(\xi, T, B^{0}\right)\right] d \xi=0 .
$$

Combining this result with that in II, 21, one concludes the

Lemma. Let $T:[\xi(u), \mathfrak{B}]$ be a continuous transformation which is $\mathrm{BV} E(T$, $\left.\mathfrak{B}^{0}\right)$. Then for any sequence of maximal systems $S_{n}(\mathfrak{B})$ for $T$ such that $\left\|S_{n}(\mathfrak{B})\right\|$ converges to zero, it is true that

$$
\int \mathrm{K}\left(\xi, T, \mathfrak{B}^{0}\right) d \xi=\lim _{n \rightarrow \infty} \sum_{B \text { in } \mathcal{S}_{n}(\mathfrak{B})} \int c\left(\xi, T, B^{0}\right) d \xi .
$$




\section{Chapter III}

\section{ON FLAT CONTINUOUS SURFACES}

1. Suppose that $S$ is a flat continuous surface (see $I, 3)$ lying in the $\xi$-plane. Every representation $[\xi(u), \mathfrak{B}]$ for $S$ determines a bounded continuous transformation $T$ from the simple Jordan region $\mathfrak{B}$ to the $\xi$-plane (see II, 16). For brevity, $[\xi(u), \mathfrak{B}]$ is termed BV $E, \mathrm{AC} E$, and so on, whenever $T$ is $\mathrm{BV} E(T$, $\left.\mathfrak{B}^{0}\right)$, AC $E\left(T, \mathfrak{B}^{0}\right)$, and so on.

LEMma. The essential multiplicity $\mathrm{K}\left(\xi, T, \mathfrak{B}^{0}\right)$ is independent of the choice of the representation $[\xi(u), \mathfrak{B}]$ for $S$.

Proof. Let $\left[\xi_{1}(u), \mathfrak{B}_{1}\right],\left[\xi_{2}(u), \mathfrak{B}_{2}\right]$ be any two representations for $S$; denote the corresponding transformations by $T_{1}, T_{2}$. From I, 1 it follows that there exists a sequence of topological maps of $\mathfrak{B}_{1}$ on to $\mathfrak{B}_{2}$ given by $\left[\bar{u}_{n}(u), \mathfrak{B}_{1}\right]$, $n=3,4, \cdots$, such that $\left\|\xi_{1}(u)-\xi_{2}\left(\bar{u}_{n}(u)\right)\right\|<n^{-1}$ for $u$ in $\mathfrak{B}_{1}$. Let $T_{n}$ denote the transformation given by $\left[\xi_{2}\left(\bar{u}_{n}(u)\right), \mathfrak{B}_{1}\right]$ for $n=3,4, \cdots$. From the definition of the essential multiplicity (see II, 2,6 ) and the nature of a topological map, it is clear that $\mathrm{K}\left(\xi, T_{2}, \mathfrak{B}_{2}^{0}\right)=\mathrm{K}\left(\xi, T_{n}, \mathfrak{B}_{1}^{0}\right)$ for $n=3,4, \cdots$. Since (see II, 1) $\lim \rho\left(T_{n}, T_{1} ; \mathfrak{B}_{1}\right)=0$, it follows from II, 2, 2 that $\lim \inf \mathrm{K}(\xi$, $\left.T_{n}, \mathfrak{B}_{1}^{0}\right) \geqq \mathrm{K}\left(\xi, T_{1}, \mathfrak{B}_{1}^{0}\right)$. Combining these relations, one obtains $\mathrm{K}\left(\xi, T_{2}, \mathfrak{B}_{2}^{0}\right)$ $\geqq \mathrm{K}\left(\xi, T_{1}, \mathfrak{B}_{1}^{0}\right)$. By symmetry, the opposite inequality follows; thus the lemma is established.

2. In view of the lemma above, one may define an essential multiplicity for a flat continuous surface $S$ lying in the $\xi$-plane by the relation $\mathrm{K}(\xi, S)$ $=\mathrm{K}\left(\xi, T, \mathfrak{B}^{0}\right)$, where $T$ is the transformation associated with any representation $[\xi(u), \mathfrak{B}]$ for $S$.

LEMMA. The essential multiplicity $\mathrm{K}(\xi, S)$ is a lower semi-continuous functional in each of its arguments $\xi$ and $S$.

Proof. The fact that $\mathrm{K}(\xi, S)$ is a lower semi-continuous function of $\xi$ follows from II, 2, 1. Next, suppose that the flat surfaces $S_{n}$ in the $\xi$-plane converge to the surface $S_{0}$. From I, 1 it follows that there exist representations $\left[\xi_{n}(u), \mathfrak{B}\right]$ for $S_{n}$, for $n=0,1,2, \cdots$, such that the corresponding transformations $T_{n}$ satisfy $\lim \rho\left(T_{n}, T_{0} ; \mathfrak{B}\right)=0$. So from II, 2, 2 follows

$$
\lim \inf \mathrm{K}\left(\xi, S_{n}\right)=\lim \inf \mathrm{K}\left(\xi, T_{n}, \mathfrak{B}^{0}\right) \geqq \mathrm{K}\left(\xi, T_{0}, \mathfrak{B}^{0}\right)=\mathrm{K}\left(\xi, S_{0}\right) .
$$

This establishes the lemma.

3. For any flat surface $S$ in the $\xi$-plane, define (see II, 2)

$$
e V(S)=\left\{\begin{array}{cl}
\int \mathrm{K}(\xi, S) d \xi & \text { if } \mathrm{K}(\xi, S) \text { is summable; } \\
+\infty & \text { otherwise. }
\end{array}\right.
$$

The quantity $e V(S)$ is termed the essential variation for the surface $S$. If 
$e V(S)$ is finite, then $S$ is said to be a surface of bounded essential variationbriefly, BEV. From the remarks in II, 12 follow the

1. Corollary. A sufficient condition that a flat surface be $\mathrm{BEV}$ is that it possess a representation which is $\mathrm{BV} E$. A necessary condition that a flat surface be $\mathrm{BEV}$ is that each of its representations be $\mathrm{BV} \mathcal{E}$. Thus if one representation for the surface is $\mathrm{BV} E$, then all representations are $\mathrm{BV} \varepsilon$.

2. Corollary. The essential variation eV(S) is a lower semi-continuous functional of $S$.

4. From the lemmas in II, 9, 12 one obtains the

1. LEMMA. If $S$ is a flat surface which is $\mathrm{BEV}$, and if $[\xi(u), \mathfrak{B}]$ is any representation for $S$, then

$$
e V(S) \geqq \int_{\mathfrak{B}^{0}}|\mathcal{F}(u, T)| d u,
$$

where $T$ is the corresponding transformation. A necessary and sufficient condition that the sign of equality hold here is that $[\xi(u), \mathfrak{B}]$ be AC $E$.

2. LEMMA. If $S$ is a flat surface which is $\mathrm{BEV}$, and if $[\xi(u), \mathfrak{B}]$ is any representation for $S$ for which the ordinary Jacobian exists almost everywhere in $\mathfrak{B}^{0}$, then

$$
e V(S) \geqq \int_{\mathscr{B} 0}|J(u, T)| d u
$$

where $T$ is the corresponding transformation. A necessary and sufficient condition that the sign of equality hold here is that $[\xi(u), \mathfrak{B}]$ be AC $E$.

5. For the purpose of comparing results with those in the literature for surfaces given in non-parametric representation, it is necessary to recall the concepts for bounded variation and absolute continuity used by Tonelli (see Tonelli [1]). Let $f(u)=f\left(u^{1}, u^{2}\right)$ be a real, single-valued function defined and continuous on the interval $\Im=[\alpha, \beta]=\left[\alpha^{1}, \beta^{1} ; \alpha^{2}, \beta^{2}\right]$ (see 4). For fixed $u^{2}$ in $\left[\alpha^{2}, \beta^{2}\right]$, denote by $V_{u^{1}}\left(f ; u^{2}\right)$ the total variation of $f\left(u^{1}, u^{2}\right)$ as a function of $u^{1}$ on $\left[\alpha^{1}, \beta^{1}\right]$. The function $V_{u^{1}}\left(f ; u^{2}\right)$ is a lower semi-continuous function of $u^{2}$ on $\left[\alpha^{2}, \beta^{2}\right]$, hence is measurable. Define $V_{u^{2}}\left(f ; u^{1}\right)$ by interchanging the roles of $u^{1}$ and $u^{2}$. If both $V_{u^{1}}\left(f ; u^{2}\right), V_{u^{2}}\left(f ; u^{1}\right)$ are summable on their respective intervals of definition, then $f(u)$ is said to be of bounded variation in the sense of Tonelli on $\Im$-briefly, BV T on $\Im$-and the total $u^{1}$ - and $u^{2}$-variations of $f(u)$ on $\Im$ are defined by

$$
V_{u 1}(f)=\int_{\alpha^{2}}^{\beta^{2}} V_{u 1}\left(f ; u^{2}\right) d u^{2}, \quad V_{u^{2}}(f)=\int_{\alpha^{1}}^{\beta^{1}} V_{u^{2}}\left(f ; u^{1}\right) d u^{1}
$$


If $f(u)$ is BV $T$ on $\Im$, then it follows that the partial derivatives $f_{u^{1}}(u), f_{u^{2}}(u)$

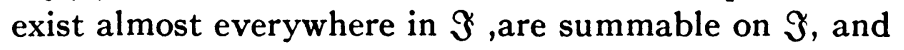

$$
\text { 1. } \quad V_{u 1}(f) \geqq \int_{\Im}\left|f_{u 1}(u)\right| d u, \quad V_{u 2}(f) \geqq \int_{\Im}\left|f_{u^{2}}(u)\right| d u .
$$

6. Assume that $f(u)$ is BV $T$ on $\Im$. If moreover, for almost every $u^{2}$ in $\left[\alpha^{2}, \beta^{2}\right]$ it is true that $f\left(u^{1}, u^{2}\right)$ is an absolutely continuous function of $u^{1}$ on $\left[\alpha^{1}, \beta^{1}\right]$, and if a similar relation holds with the roles of $u^{1}$ and $u^{2}$ reversed, then $f(u)$ is said to be absolutely continuous in the sense of Tonelli on $\Im-$ briefly, AC $T$ on $\Im$. A necessary and sufficient condition that a function $f(u)$ which is BV $T$ on $\Im$ be AC $T$ on $\Im$ is that the sign of equality hold in both of the relations III, $\mathbf{5}, 1$.

7. Let $f(u)$ be a real, single-valued function defined and continuous on the interval $\Im=[\alpha, \beta]$. Consider the continuous transformation defined by

$$
{ }^{1} T: \quad \xi^{1}=u^{2}, \quad \xi^{2}=f\left(u^{1}, u^{2}\right), \quad\left(u^{1}, u^{2}\right) \text { in } \Im .
$$

Notice that for a fixed $u^{2}=\gamma^{2}$ in $\left[\alpha^{2}, \beta^{2}\right],{ }^{1} T$ gives a linear transformation from the closed linear interval $u^{2}=\gamma^{2}, \alpha^{1} \leqq u^{1} \leqq \beta^{1}$ to a bounded portion of the line $\xi^{1}=\gamma^{2}$. For this transformation it is known (see Rado [4]) that a necessary and sufficient condition that $f\left(u^{1}, \gamma^{2}\right)$ be of bounded variation on $\left[\alpha^{1}, \beta^{1}\right]$ is that $N\left(\left(\gamma^{2}, \xi^{2}\right),{ }^{1} T, \Im^{0}\right)$ be summable as a function of $\xi^{2}$ (see $4 ;$ II, 1 ), and that if $f\left(u, \gamma^{2}\right)$ is of bounded variation on $\left[\alpha^{1}, \beta^{1}\right]$ then (see III, 5)

$$
\int N\left(\left(\gamma^{2}, \xi^{2}\right),{ }^{1} T, \Im^{0}\right) d \xi^{2}=V_{u 1}\left(f ; \gamma^{2}\right), \quad \alpha^{2} \leqq \gamma^{2} \leqq \beta^{2} .
$$

Thus it follows that if $V_{u^{1}}\left(f ; u^{2}\right)$ is summable on $\left[\alpha^{2}, \beta^{2}\right]$, then $\int N\left(\left(\xi^{1}, \xi^{2}\right)\right.$, $\left.{ }^{1} T, \Im^{0}\right) d \xi^{2}$ is a summable function of $\xi^{1}$ on $\left[\alpha^{2}, \beta^{2}\right]$. Now (see $\mathrm{R}^{2}[1]$, chap. III]) $N\left(\xi,{ }^{1} T, \Im^{0}\right)$ is measurable in the $\xi$-plane. So if $V_{u^{1}}\left(f ; u^{2}\right)$ is summable on $\left[\alpha^{2}, \beta^{2}\right]$, it follows from the theorem of Fubini that $N\left(\xi,{ }^{1} T, \Im^{0}\right)$ is summable, hence (see II, 4) ${ }^{1} T$ is BV $\Im^{0}$, and (see III, 5)

2.

$$
\begin{aligned}
\int N\left(\xi,{ }^{1} T, \Im^{0}\right) d \xi & =\int d \xi^{1} \int N\left(\left(\xi^{1}, \xi^{2}\right),{ }^{1} T, \Im^{0}\right) d \xi^{2} \\
& =\int_{\alpha^{2}}^{\beta^{2}} V_{u 1}\left(f ; u^{2}\right) d u^{2}=V_{u^{1}}(f) .
\end{aligned}
$$

Conversely, suppose that ${ }^{1} T$ is $\mathrm{BV} \mathcal{Y}^{0}$; then (see II, 4) it is true that $N\left(\xi,{ }^{1} T\right.$, $\left.\Im^{0}\right)$ is summable, so that by the theorem of Fubini, the function $N\left(\left(\gamma^{2}, \xi^{2}\right)\right.$, $\left.{ }^{1} T, \Im^{0}\right)$ is summable for almost every choice of $\xi^{1}=\gamma^{2}$ in the interval $\left[\alpha^{2}, \beta^{2}\right]$, and

3. $\quad \int N\left(\xi,{ }^{1} T, \Im^{0}\right) d \xi=\int d \xi^{1} \int N\left(\left(\xi^{1}, \xi^{2}\right),{ }^{1} T, \Im^{0}\right) d \xi^{2}$. 
By the result cited above, it follows that $f\left(u^{1}, \gamma^{2}\right)$ is of bounded variation on $\left[\alpha^{1}, \beta^{1}\right]$ and relation 1 holds for almost every choice of $u^{2}=\gamma^{2}$ in $\left[\alpha^{2}, \beta^{2}\right]$. In view of relation 3 , it follows that $V_{u^{1}}\left(f ; u^{2}\right)$ is a summable function of $u^{2}$ on $\left[\alpha^{2}, \beta^{2}\right]$. A similar reasoning applies to the continuous transformation defined by

$$
{ }^{2} T: \quad \xi^{1}=u^{1}, \quad \xi^{2}=f\left(u^{1}, u^{2}\right), \quad\left(u^{1}, u^{2}\right) \text { in } \Im .
$$

These results are summarized in the

LEMMA. Let $f(u)=f\left(u^{1}, u^{2}\right)$ be a real, single-valued function defined and continuous on the interval $\Im$. Consider the two transformations

$$
\begin{aligned}
& { }^{1} T: \quad \xi^{1}=u^{2}, \quad \xi^{2}=f\left(u^{1}, u^{2}\right), \quad\left(u^{1}, u^{2}\right) \text { in } \Im \text {; } \\
& { }^{2} T: \quad \xi^{1}=u^{1}, \quad \xi^{2}=f\left(u^{1}, u^{2}\right), \quad\left(u^{1}, u^{2}\right) \text { in } \Im .
\end{aligned}
$$

$A$ necessary and sufficient condition that $f(u)$ be $\mathrm{BV} T$ on $\Im$ is that both ${ }^{1} T$ and

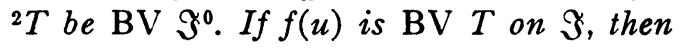

$$
\begin{aligned}
& \int N\left(\xi,{ }^{1} T, \Im^{0}\right) d \xi=V_{u^{1}}(f) \geqq \int_{\Im}\left|f_{u^{1}}(u)\right| d u ; \\
& \int N\left(\xi,{ }^{2} T, \Im^{0}\right) d \xi=V_{u^{2}}(f) \geqq \int_{\Im}\left|f_{u^{2}}(u)\right| d u .
\end{aligned}
$$

$A$ necessary and sufficient condition that the sign of equality hold in both these relations is that $f(u)$ be AC $T$ on $\Im$.

8. Again let $f(u)$ be a real, single-valued function defined and continuous on the interval $\Im=[\alpha, \beta]$. Retain the notation of the preceding section. For an interval $I=\left[\gamma^{1}, \delta^{1} ; \gamma^{2}, \delta^{2}\right]$ contained in $\Im$ define

$$
\psi(I)=\int_{\gamma^{2}}^{\delta^{2}}\left|f\left(\delta^{1}, u^{2}\right)-f\left(\gamma^{1}, u^{2}\right)\right| d u^{2} .
$$

It will now be shown that for any interval system $S(\Im)$ (see 4),

$$
\psi(S(\Im)) \leqq \int \mathrm{K}\left(\xi,{ }^{1} T, \Im^{0}\right) d \xi
$$

provided that $\mathrm{K}\left(\xi^{1}, T, \Im\right)$ is summable. Evidently ${ }^{1} \psi(I)$ is the measure of the set of points $\xi=\left(\xi^{1}, \xi^{2}\right)$ which satisfy the inequalities

$$
\left(f\left(\delta^{1}, u^{2}\right)-\xi^{2}\right)\left(f\left(\gamma^{1}, u^{2}\right)-\xi^{2}\right)<0, \quad \gamma^{2}<\xi^{1}=u^{2}<\delta^{2} .
$$

Consider any point $\xi_{0}=\left(\xi_{0}^{1}, \xi_{0}^{2}\right)$ which satisfies these inequalities. Its inverse ${ }^{1} T^{-1}\left(\xi_{0}\right)$ evidently is contained on the line $u^{2}=\xi_{0}^{1}$. Since $f\left(u^{1}, \xi_{0}^{1}\right)$ is continuous on $\left[\gamma^{1}, \delta^{1}\right]$ and has opposite signs at the points $u^{1}=\gamma^{1}, u^{2}=\delta^{1}$, it follows that $\xi_{0}$ has a model in the interior of $I$, and that $\mu\left(\xi_{0},{ }^{1} T, I\right)= \pm 1$. From II, 6 
it follows that $\mathrm{K}\left(\xi_{0},{ }^{1} T, I^{0}\right) \geqq 1$. Thus ${ }^{1} \psi(I) \leqq \int \mathrm{K}\left(\xi, T, I^{0}\right) d \xi$. From II, 19, 2 it follows that

$$
{ }^{\mathcal{L}} \psi(S(\Im)) \leqq \sum_{I \text { in } S(\Im)} \int \mathrm{K}\left(\xi, T, I^{0}\right) d \xi \leqq \int \mathrm{K}\left(\xi, T, \Im^{0}\right) d \xi .
$$

Thus relation 1 is established. Now consider the sequence of subdivisions $S_{m}(\Im)$ of $\Im$ into intervals $\left[\gamma_{j-1}^{1}, \gamma_{j}^{1} ; \alpha^{2}, \beta^{2}\right]$, where $\gamma_{j}^{1}=\alpha^{1}+\left(\beta^{1}-\alpha^{1}\right) j / m, j=0$, $\cdots, m$. Evidently

$$
{ }^{1} \psi\left(S_{m}(\Im)\right)=\int_{\alpha^{2}}^{\beta^{2}} \sum_{j=1}^{m}\left|f\left(\gamma_{j}^{1}, u^{1}\right)-f\left(\gamma_{j-1}^{1} ; u^{2}\right)\right| d u^{2}
$$

and (see III, 5)

$$
\lim \sum_{j=1}^{m}\left|f\left(\gamma_{j}^{1}, u^{2}\right)-f\left(\gamma_{j-1}^{1}, u^{2}\right)\right|=V_{u 1}\left(f ; u^{2}\right) .
$$

From the lemma of Fatou it follows that $V_{u^{1}}\left(f ; u^{2}\right)$ is summable on $\left[\alpha^{2}, \beta^{2}\right]$ whenever $\mathrm{K}\left(\xi,{ }^{1} T, \Im^{0}\right)$ is summable, and

$$
V_{u 1}(f) \leqq \int \mathrm{K}\left(\xi,{ }^{1} T, \Im^{0}\right) d \xi .
$$

Since $\mathrm{K}\left(\xi,{ }^{1} T, \Im^{0}\right) \leqq N\left(\xi,{ }^{1} T, \Im^{0}\right)$, it is clear from III, 7 that a necessary and sufficient condition that $V_{u^{1}}\left(f ; u^{2}\right)$ be summable on $\left[\alpha_{2}, \beta_{2}\right]$ is that $K\left(\xi,{ }^{1} T, \Im^{0}\right)$ be summable. If $\mathrm{K}\left(\xi,{ }^{1} T, \Im^{0}\right)$ is summable, then

$$
V_{u 1}(f)=\int \mathrm{K}\left(\xi,{ }^{1} T, \Im^{0}\right) d \xi \geqq \int_{\Im}\left|f_{u 1}(u)\right| d u .
$$

Similar statements are valid for the transformation ${ }^{2} T$. Combining these facts with those in III, $3,4,7$, one obtains the

LEMMA. Let $f(u)=f\left(u^{1}, u^{2}\right)$ be a real, single-valued function defined and continuous on the interval $\Im$. Consider the flat surfaces having the representations

$$
{ }^{1} S: \quad\left[\left(u^{2}, f\left(u^{1}, u^{2}\right)\right), \Im\right] ; \quad{ }^{2} S: \quad\left[\left(u^{1}, f\left(u^{1}, u^{2}\right)\right), \Im\right] .
$$

$A$ necessary and sufficient condition that $f(u)$ be BV $T$ on $\Im$ is that both ${ }^{1} S$ and ${ }^{2} S$ be BEV. If $f(u)$ is BV $T$ on $\Im$, then

$$
e V\left({ }^{1} S\right)=V_{u 1}(f) \geqq \int_{\Im}\left|f_{u 1}(u)\right| d u ; \quad e V\left({ }^{2} S\right)=V_{u^{2}}(f) \geqq \int_{\Im}\left|f_{u^{2}}(u)\right| d u .
$$

A necessary and sufficient condition that the sign of equality hold in both these relations is that $f(u)$ be AC $T$ on $\Im$. A necessary and sufficient condition that $f(u)$ be AC $T$ on $\Im$ is that both of the representations for ${ }^{1} S$ and ${ }^{2} S$ be AC $E$. 


\section{Chapter IV}

\section{ON THE ESSENTIAL AREA}

1. Let $S$ be a continuous surface in $x$-space (see $I, 1$ ), and let $[x(u), \mathfrak{B}]$ be any representation for $S$. With $S$ there is associated the three projection surfaces ${ }^{i} S$ upon the coordinate planes ${ }^{i} x$ having representations $\left[{ }^{i} x(u), \mathfrak{B}\right]$, which determine bounded continuous transformations ${ }^{i} T$ for $i=1,2,3$ (see $4 ; I, 4)$. In this chapter, the theory of the preceding chapters is used when the plane $\xi$ coincides in turn with the coordinate planes ${ }^{i} x, i=1,2,3$. The following triple notation is useful (see III, 2)

$$
\begin{aligned}
\mathrm{K}(x, S) & \equiv\left(\mathrm{K}\left({ }^{1} x,{ }^{1} S\right), \mathrm{K}\left({ }^{2} x,{ }^{2} S\right), \mathrm{K}\left({ }^{3} x,{ }^{3} S\right)\right) ; \\
\mathcal{f}(u,[x, \mathfrak{B}]) & \equiv\left(\mathcal{F}\left(u,{ }^{1} T\right), \mathcal{F}\left(u,{ }^{2} T\right), \mathcal{F}\left(u,{ }^{3} T\right)\right) \text { for } u \text { in } \mathfrak{B}^{0} ; \\
J(u,[x, \mathfrak{B}]) & \equiv\left(J\left(u,{ }^{1} T\right), J\left(u,{ }^{2} T\right), J\left(u,{ }^{3} T\right)\right) \text { for } u \text { in } \mathfrak{B}^{0} .
\end{aligned}
$$

The triple $[x(u), \mathfrak{B}]$ is said to be $\mathrm{BV} E, \mathrm{AC} E$, and so on, when each of the associated transformations ${ }^{i} T$ is $\mathrm{BV} E\left({ }^{i} T, \mathfrak{B}^{0}\right), \mathrm{AC} E\left({ }^{i} T, \mathfrak{B}^{0}\right)$, and so on, for $i=1,2,3$ (cf. III, 1).

2. Given a continuous surface $S$ for which $\mathrm{K}(x, S)$ is summable, that is, for which each of the projection surfaces ${ }^{1} S,{ }^{2} S,{ }^{3} S$ is BEV (see III, 3). If $[x(u), \mathfrak{B}]$ be any representation for $S$, define, for any simple Jordan region $B$ in $\mathfrak{B}$

$$
\begin{array}{rlrl}
{ }^{i} \phi(B) & =\int \mathrm{K}\left({ }^{i} x,{ }^{i} T, B^{0}\right) d^{i} x, & i=1,2,3 ; \\
\phi(B) & =\left({ }^{1} \phi(B),{ }^{2} \phi(B),{ }^{3} \phi(B)\right), & \Phi(B)=\|\phi(B)\| & \text { for } \quad B \text { in } \mathfrak{B} .
\end{array}
$$

It follows from II, 19 that, for any finite system $S(\mathfrak{B})$ of nonoverlapping simple Jordan regions in $\mathfrak{B}$ (see 4 ), it is true that ${ }^{i} \phi(S(\mathfrak{B})) \leqq{ }^{i} \phi(\mathfrak{B})$ for $i=1,2,3$. Thus one has (see III 1-3)

1.

$$
e V(i S)={ }^{i} \phi(\mathfrak{B})=U\left(\mathfrak{B} ;\left[{ }^{i} \phi, \mathfrak{B}\right]\right) \quad \text { for } i=1,2,3 .
$$

From a remark in 5 follows the fact that $U(\mathfrak{B} ;[\Phi, \mathfrak{B}])$ is finite; moreover, one has the

LEMma. The quantity $U(\mathfrak{B} ;[\Phi, \mathfrak{B}])$ is independent of the choice of the representation for $S$.

Proof. Let $\left[x_{1}(u), \mathfrak{B}_{1}\right],\left[x_{2}(u), \mathfrak{B}_{2}\right]$ be any two representations for $S$. From I, 1 it follows that there exists a sequence of topological maps of $\mathfrak{B}_{1}$ onto $\mathfrak{B}_{2}$ given by $\left[\bar{u}_{n}(u), \mathfrak{B}_{1}\right]$ for $n=3,4, \cdots$ such that $\left\|x_{1}(u)-x_{2}\left(\bar{u}_{n}(u)\right)\right\|<n^{-1}$ for $u$ in $\mathfrak{B}_{1}$. Denote by ${ }^{i} T_{n}$ the transformations given by the triples $\left[{ }^{i} x_{2}\left(\bar{u}_{n}(u)\right)\right.$, $\left.\mathfrak{B}_{1}\right]$ for $n=3,4, \cdots ; i=1,2,3$. Put

$$
{ }^{i} \phi_{n}(B)=\int \mathrm{K}\left({ }^{i} x,{ }^{i} T_{n}, B^{0}\right) d^{i} x, \quad i=1,2,3 ; n=1,2,3, \cdots ;
$$




$$
\phi_{n}(B)=\left({ }^{1} \phi_{n}(B),{ }^{2} \phi_{n}(B),{ }^{3} \phi_{n}(B)\right), \quad \Phi_{n}(B)=\left\|\phi_{n}(B)\right\| \quad \text { for } \quad B \text { in } \mathfrak{B} .
$$

To any finite system $S(\mathfrak{B})$ (see 4 ) there corresponds under the map $\left[\bar{u}_{n}(u)\right.$, $\mathfrak{B}_{1}$ ] a finite system $S_{n}\left(\mathfrak{B}_{2}\right)$. Clearly (see III, 1)

$$
U\left(\mathfrak{B}_{2},\left[\Phi_{2}, \mathfrak{B}_{2}\right]\right) \geqq \Phi_{2}\left(S_{n}\left(\mathfrak{B}_{2}\right)\right)=\Phi_{n}\left(S\left(\mathfrak{B}_{1}\right)\right) \quad \text { for } n=3,4, \cdots .
$$

Since $\lim \rho\left({ }^{i} T_{n},{ }^{i} T_{1} ; \mathfrak{B}_{1}\right)=0$ for $i=1,2,3$, it follows from II , 2, 2 and the lemma of Fatou that $\lim$ inf $\Phi_{n}\left(S\left(\mathfrak{B}_{1}\right)\right) \geqq \Phi_{1}\left(S\left(\mathfrak{B}_{1}\right)\right)$. From the preceding relations and the definition of the $U$-function (see 4), it follows that $U\left(\mathfrak{B}_{2} ;\left[\Phi_{2}, \mathfrak{B}_{2}\right]\right.$ ) $\geqq U\left(\mathfrak{B}_{1} ;\left[\Phi_{1}, \mathfrak{B}_{1}\right]\right)$. The opposite inequality follows by symmetry, and the lemma is established.

3. Let $S$ be a continuous surface in $x$-space. In view of the preceding lemma, one may define the essential area $e A(S)$ for the surface as follows. If $[x(u), \mathfrak{B}]$ be any representation for $S$, set

$$
e A(S)=\left\{\begin{array}{cl}
U(\mathfrak{B} ;[\Phi, \mathfrak{B}]) & \text { if } \mathrm{K}(x, S) \text { is summable } \\
+\infty & \text { otherwise. }
\end{array}\right.
$$

Clearly $e A(S)$ is independent of the choice of the representation for $S$, although it is not clear as to whether it is also independent of the choice of a coordinate system in $x$-space. If $S$ is a flat surface in a coordinate plane (see I, 3), then $e A(S)=e V(S)$ (see III, 2; IV, 2, 1)( ${ }^{8}$ ).

4. From $5 ; \mathrm{IV}, 2,1$ follows the

THEOREM. $A$ necessary and sufficient condition that the essential area $e A(S)$ of a surface $S$ be finite is that each of the projection surfaces ${ }^{1} S,{ }^{2} S,{ }^{3} S$ be of bounded essential variation. Between the essential area and the essential variations of the projection surfaces, the following relation exists

$$
e V(i S) \leqq e A(S) \leqq \sum_{i=1}^{3} e V(i S)
$$

Notice that this theorem is an analogue for continuous surfaces of the result for continuous curves cited in 1,1 .

5. LEMma. The essential area $\mathrm{eA}(S)$ is a lower semi-continuous functional of $S$.

Proof. Suppose the sequence of continuous surfaces $S_{n}$ converges to a surface $S_{0}$; from I, 1 it follows that there exist representations $\left[x_{n}(u), \mathfrak{B}\right]$ for $n=0,1,2, \cdots$ such that $x_{n}(u)$ converges on $\mathfrak{B}$ uniformly to $x_{0}(u)$. Adopt the notation of IV, 2, using a subscript $n$ to distinguish the functions belonging to

(8) Since $e V(i S)=e A(i S)$ for $i=1,2,3$, the notion of essential variation might very well be discarded. However, it has not been the custom to speak of the length of a one-dimensional curve, but rather to speak of the total variation of a function representing that curve. To preserve this parallel between the theory of curves and the theory of surfaces, the concept of essential variation has been introduced here. 
$S_{n}$. From II, 2, 2 and the lemma of Fatou, it follows that lim inf ${ }^{i} \phi_{n}(B)$ $\geqq \phi_{0}(B)$ for $B$ in $B, i=1,2,3$. From the lemma in 5 and the definition of the essential area follows lim inf $e A\left(S_{n}\right) \geqq e A\left(S_{0}\right)$, and the lemma is proved.

6. THEOREM. If the essential area for a surface $S$ is finite, and if $[x(u), \mathfrak{B}]$ be any representation for $S$, then the triple $\mathcal{F}(u,[x, \mathfrak{B}])$ of generalized Jacobians exists almost everywhere in $\mathfrak{B}^{0}$, is summable on $\mathfrak{B}^{0}$, and

1.

$$
e A(S) \geqq \int_{\mathfrak{B} 0}\|\mathcal{F}(u,[x, \mathfrak{B}])\| d u .
$$

$A$ sufficient condition that the sign of equality hold here is that the triple $[x(u), \mathfrak{B}]$ be AC E; a necessary condition that the sign of equality hold is that the triple be $\mathrm{AC} \varepsilon$.

Proof. Since $e A(S)$ is finite, it is true that each is is BEV (see IV, 4), hence $\mathrm{K}\left({ }^{i} x,{ }^{i} T, \mathfrak{B}^{0}\right)$ is summable for $i=1,2,3$ (see III, 2-3). Thus (see IV, $2 ;$ II , 9, 1) each $\mathcal{F}(u, i T)$ exists almost everywhere in $\mathfrak{B}^{0}$, is summable on $\mathfrak{B}^{0}$, and

2.

$$
{ }^{i} \phi(B) \geqq \int_{B^{0}}\left|\mathcal{F}\left(u,{ }^{i} T\right)\right| d u \text { for } B \text { in } \mathfrak{B}, \quad i=1,2,3 .
$$

Define

3. $\quad \psi(B)=\int_{B^{0}}\left|\mathcal{F}\left(u,{ }^{i} T\right)\right| d u, \quad i=1,2,3$;

$$
\psi(B)=\left({ }^{1} \psi(B),{ }^{2} \psi(B),{ }^{2} \psi(B)\right), \quad \Psi(B)=\|\psi(B)\| \quad \text { for } \quad B \text { in } \mathfrak{B} .
$$

If $S(\mathscr{B})$ be any finite system, it follows by a known inequality (see Hardy, Littlewood, Polya [1, chap. VI]) that

Thus

$$
\Psi(S(\mathfrak{B})) \leqq \int_{\mathfrak{B}^{0}}\|\mathcal{F}(u,[x, \mathfrak{B}])\| d u .
$$

4.

$$
U(\mathfrak{B} ;[\Psi, \mathfrak{B}]) \leqq \int_{\mathfrak{B}^{\mathfrak{b}}}\|\mathcal{F}(u,[x, \mathfrak{B}])\| d u .
$$

On the other hand, let $R$ be a simple Jordan region in $\mathscr{B}$ which admits of an interval subdivision. Then it is true (see $R^{2}[2$, chap. II, \$10]) that there exists a sequence of interval subdivisions $S_{n}(R)$ such that

Thus

$$
\lim \Psi\left(S_{n}(R)\right)=\int_{R^{0}}\|\mathcal{F}(u,[x, \mathfrak{B}])\| d u .
$$

5.

$$
U(\mathfrak{B} ;[\Psi, \mathfrak{B}]) \geqq \int_{\mathbb{R}^{0}}\|\mathcal{F}(u,[x, \mathfrak{B}])\| d u .
$$


Since the open domain $\mathfrak{B}^{\circ}$ may be filled up from the interior (see 4) by a sequence of simple regions of type $R$ (see Kerékjártó [1]), one concludes from relations 4 and 5 that

6.

$$
U(\mathfrak{B} ;[\Psi, \mathfrak{B}])=\int_{\mathfrak{B}^{0}}\|\mathcal{F}(u,[x, \mathfrak{B}])\| d u .
$$

Relations 2 and 3 imply

7.

$$
U(\mathfrak{B} ;[\Phi, \mathfrak{B}]) \geqq U(\mathfrak{B} ;[\Psi, \mathfrak{B}]) .
$$

In view of IV, 3, the first statement in the theorem is established. Now (see II, 9, 12) if $[x(u), \mathfrak{B}]$ is AC $E$, then the sign of equality holds in 2 , hence in 7 . Thus a sufficient condition that the sign of equality hold in 1 is that $[x(u), \mathfrak{B}]$ be AC $E$. Next, suppose the sign of equality holds in relation 1 . Let $I$ be any interval in $\mathfrak{B}$; extend the lines forming the boundary of $I$ indefinitely. The simple Jordan region $\mathfrak{B}$ is thus divided into a possibly enumerable number of simple Jordan regions $I \equiv B_{0}, B_{1}, B_{2}, \ldots$. For any simple Jordan region $B$ in $\mathscr{B}$, the reasoning leading to relation 6 gives

$$
U(B ;[\Psi, \mathfrak{B}])=\int_{B^{0}}\|\mathcal{F}(u,[x, \mathfrak{B}])\| d u .
$$

Since a bounded portion of a straight line is rectifiable, it follows that

$$
U(\mathfrak{B} ;[\Psi, \mathfrak{B}])=\sum_{n} U\left(B_{n} ;[\Psi, \mathfrak{B}]\right) \text {. }
$$

From this relation and relations 2,3 , one finds

$$
U(\mathfrak{B} ;[\Phi, \mathfrak{B}]) \geqq \sum_{n} U\left(B_{n} ;[\Phi, \mathfrak{B}]\right) \geqq \sum_{n} U\left(B_{n} ;[\Psi, \mathfrak{B}]\right)=U(\mathfrak{B} ;[\Psi, \mathfrak{B}]) .
$$

But since the sign of equality holds in 1 by hypothesis, it follows that the sign of equality must hold at every step in the preceding inequalities. In particular, then

$$
U(I ;[\Phi, \mathfrak{B}])=U(I ;[\Psi, \mathfrak{B}])=\int_{I}\|\mathcal{F}(u,[x, \mathfrak{B}])\| d u .
$$

If $s$ be any closed oriented square in $\mathfrak{B}$, then obviously (see II, 3-8; IV, 1-3)

$$
\begin{aligned}
G\left(s,{ }^{i} T, \mathcal{E}\left({ }^{i} T, \mathfrak{B}^{0}\right)\right) & \leqq \int \mathrm{K}\left({ }^{i} x,{ }^{i} T, s^{0}\right) d^{i} x \leqq U(s ;[\Phi, \mathfrak{B}]) \\
& =\int\|\mathcal{F}(u,[x, \mathfrak{B}])\| d u .
\end{aligned}
$$

Thus each ${ }^{i} T$ is $A C \mathcal{E}\left({ }^{i} T, \mathfrak{B}^{0}\right)$ for $i=1,2,3$-that is, $[x(u), \mathfrak{B}]$ is $\mathrm{AC} \mathcal{E}$. This completes the proof. 
7. CoRollary. If a surface $S$ has a representation $[x(u), \mathfrak{B}]$ which is $\mathrm{BV} E$, then the essential area $e A(S)$ is finite, the triple $\mathcal{F}(u,[x, \mathfrak{B}])$ of generalized Jacobians exists almost everywhere in $\mathfrak{B}^{\circ}$, is summable on $\mathfrak{B}^{0}$, and

$$
e A(S) \geqq \int_{\mathfrak{B}^{0}}\|\mathcal{F}(u,[x, \mathfrak{B}])\| d u .
$$

$A$ necessary and sufficient condition that the sign of equality hold here is that $[x(u), \mathfrak{B}]$ be AC $E$.

A proof follows from results in II, 11;III, 3, 1; IV, 4, 6. This is an analogue to the theorems in 1, 2, 3 for continuous curves; all representations for continuous curves automatically satisfy the analogue of $\mathrm{BV} E$ in one dimension whenever the length of the curves is finite.

8. Corollary. Let $S_{n}, n=0,1,2, \cdots$, be a sequence of continuous surfaces satisfying the following conditions: the surfaces $S_{n}$ converge to $S_{0}($ see $I, 1)$; each of the surfaces has a finite essential area $e A\left(S_{n}\right)$ for $n=0,1,2, \cdots$ (see $I V, 3,4)$; the surface $S_{0}$ has a representation $[x(u), \mathfrak{B}]$ for which the essential areas $e A\left(S_{n}\right)$ converge to $\int_{\mathfrak{B O}}\|\mathcal{F}(u,[x, \mathfrak{B}])\| d u$. Then the representation $[x(u), \mathfrak{B}]$ is $\mathrm{AC} \varepsilon$.

Proof. From IV, 5, 6 and the assumptions, one obtains

$$
\int_{\mathfrak{B}^{0}}\|\mathcal{F}(u,[x, \mathfrak{B}])\| d u=\lim e A\left(S_{n}\right) \geqq e A\left(S_{0}\right) \geqq \int_{\mathfrak{B}_{0}}\|\mathcal{F}(u,[x, \mathfrak{B}])\| d u .
$$

Thus the sign of equality holds throughout, and the conclusion now follows from the last part of the theorem in IV, 6 .

9. By the principle stated in I, 8, the theorem in IV, 6 may be given the following variant form.

THEOREM. A necessary condition that a representation $[x(u), \mathfrak{B}]$ for a surface $S$ be absolutely continuous $(e A, \mathcal{F})$, where $e A(S)$ is the essential area of $S$ and $\mathcal{F}(u,[x, \mathfrak{B}])$ is the triple of generalized Jacobians, is that $[x(u), \mathfrak{B}]$ be AC $\mathcal{E}$. $A$ sufficient condition that this representation for $S$ be absolutely continuous $(e A, \mathcal{F})$ is that it be AC $E$; then each of the corresponding representations for the projection surfaces ${ }^{i} S$ is also absolutely continuous $(e A, \mathcal{F})$ for $i=1,2,3$.

10. The results in IV, 6-9 are paralleled by similar theorems involving the essential area and the triple of ordinary Jacobians; one need but replace II, 9,1 by II, 9,2 in making the proofs.

THEOREM. If the essential area for a surface $S$ is finite, and if $[x(u), \mathfrak{B}]$ be any representation for $S$ for which the triple $J(u,[x, \mathfrak{B}])$ of ordinary Jacobians exists almost everywhere in $\mathfrak{B}^{0}$, then the triple is summable on $\mathfrak{B}^{0}$, and

$$
e A(S) \geqq \int_{\mathfrak{B} \mathbb{0}}\|J(u,[x, \mathfrak{B}])\| d u \text {. }
$$


$A$ sufficient condition that the sign of equality hold here is that the triple $[x(u) \mathfrak{B}]$ be AC E; a necessary condition that the sign of equality hold is that the triple be $\mathrm{AC} \varepsilon$.

11. CoROllaRy. If a surface $S$ has a representation $[x(u), \mathfrak{B}]$ which is BV $E$ and for which the triple $J(u,[x, \mathfrak{B}])$ of ordinary Jacobians exists almost everywhere in $\mathfrak{B}^{0}$, then the essential area is finite, the triple $J(u,[x, \mathfrak{B}])$ is summable on $\mathfrak{B}^{0}$, and

$$
e A(S) \geqq \int_{\mathfrak{B}^{0}}\|J(u,[x, \mathfrak{B}])\| d u .
$$

$A$ necessary and sufficient condition that the sign of equality hold here is that $[x(u), \mathfrak{B}]$ be AC $E$.

12. Corollary. Let $S_{n}, n=0,1,2, \cdots$, be a sequence of continuous surfaces satisfying the following conditions: the surfaces $S_{n}$ converge to $S_{0}$; each of the surfaces has a finite essential area $e A\left(S_{n}\right)$ for $n=0,1,2, \cdots$; the surface $S$. has a representation $[x(u), \mathfrak{B}]$ for which the triple $J(u,[x, \mathfrak{B}])$ of ordinary Jacobians exists almost everywhere in $\mathfrak{B}^{0}$ and the essential areas $e A(S)$ converge to $\int_{\mathfrak{B} O}\|J(u,[x, \mathfrak{B}])\| d u$. Then the representation $[x(u), \mathfrak{B}]$ is $\mathrm{AC} \varepsilon$.

13. Here is a variant form for the theorem in IV, 10.

THEOREM. A necessary condition that a representation $[x(u), \mathfrak{B}]$ for a surface $S$, for which the triple $J(u,[x, \mathfrak{B}])$ of ordinary Jacobians exists almost everywhere in $\mathfrak{B}^{0}$, be absolutely continuous $(e A, J)$ is that $[x(u), \mathfrak{B}]$ be $\mathrm{AC} \varepsilon$. $A$ sufficient condition that this representation for $S$ be absolutely continuous $(e A, J)$ is that it be AC $E$; then each of the corresponding representations for the projection surfaces ${ }^{~} S$ is also absolutely continuous $(e A, J)$ for $i=1,2,3$.

14. Rado (see Rado [2]) has shown that a representation $[x(u), \mathfrak{B}]$ for a surface $S$ which satisfies a Lipschitz condition of the form $\left\|x\left(u_{1}\right)-x\left(u_{2}\right)\right\|$ $\leqq L\left\|u_{1}-u_{2}\right\|$ where $L$ is a constant, is absolutely continuous $(A, J)$, where $A(S)$ is the Lebesgue area of $S$ (see $\mathrm{I}, 6)$ and $J(u,[x, \mathfrak{B}]$ ) is the triple of ordinary Jacobians. Now the representation $[x(u), \mathfrak{B}]$ is also AC $E$ (see II, 8). From the theorem in IV, 13 one concludes that the essential area and the Lebesgue area of $S$ are equal. In particular, $e A(P)=A(P)$ for every polyhedron $P$ (see I, 5).

Given any continuous surface $S$, there exists a sequence of polyhedra $P_{n}$ such that $P_{n}$ converge to $S$ and the Lebesgue areas $A\left(P_{n}\right)$ converge to $A(S)$ (see I, 6, 2). Since $e A(P)=A\left(P_{n}\right)$, one finds by using the lemma in IV, 5 the

THEOREM. The essential area of a surface $S$ does not exceed the Lebesgue area -that is, $e A(S) \leqq A(S)$. A necessary and sufficient condition that the essential area and the Lebesgue area of a surface $S$ be equal is that there exists a sequence of polyhedra $P_{n}$ such that $P_{n}$ converges to $S$ and $e A\left(P_{n}\right)$ converges to $e A(S)$. 
This theorem and the theorem in IV, 10 have the

Corollary. A sufficient condition that the Lebesgue area and the essential area of a surface $S$ be equal is that $S$ possess a representation $[x(u), \mathfrak{B}]$ which is absolutely continuous $(A, J)$, where $A(S)$ is the Lebesgue area of $S$ and $J(u$, $[x, \mathfrak{B}])$ is the triple of ordinary Jacobians.

15. In order to compare these results with those of Geöcze and Tonelli (cf. 2), and to give a proof for the statement made in I, 7 the following result is needed.

LEMMA. If a surface $S$ has a representation $[x(u), \Im]$ of non-parametric origin

$$
S: \quad x(u)=\left(u^{1}, u^{2} ; x^{3}\left(u^{1}, u^{2}\right)\right), \quad u=\left(u^{1}, u^{2}\right) \text { in } \Im,
$$

then $e A(S)=A_{*}(S)=A(S)$.

Proof. Define, for any interval $I=\left[\gamma^{1}, \delta^{1} ; \gamma^{2}, \delta^{2}\right]$ in $\Im\left({ }^{9}\right)$.

$$
\begin{aligned}
\psi(I)=\left(\int_{\gamma^{2}}^{\delta^{2}}\left|x^{3}\left(\gamma^{1}, u^{2}\right)-x^{3}\left(\delta^{1}, u^{2}\right)\right| d u^{2},\right. \\
\left.\qquad \int_{\gamma^{1}}^{\delta^{1}}\left|x^{3}\left(u^{1}, \gamma^{2}\right)-x^{3}\left(u^{2}, \delta^{2}\right)\right| d u^{1},|I|\right), \quad \Psi(I)=\|\psi(I)\| .
\end{aligned}
$$

If ${ }^{i} T, i=1,2,3$, be the associated continuous transformations (see IV, 1), then the reader will observe that the transformations ${ }^{1} T$ and ${ }^{2} T$ bear the same relation to the function $x^{3}(u)$ as those in III, 7,8 bear to the function $f(u)$ therein considered; the transformation ${ }^{3} T$ is simply the identity mapping of $\Im$ in the $u$-plane onto a congruent interval in the ${ }^{3} x$-plane. Define a triple of $b$-functions $[\phi, \Im]$ as in IV, 2. The reasoning in III, 8 shows that $\psi(I) \leqq \phi(I)$ for $I$ in. $\Im$. Now Rado has shown that (see Rado [1])

$$
A_{*}(S)=\text { l.u.b. } \Psi(S(\Im)) \text { for interval subdivisions } S(\Im) \text {. }
$$

From these relations it is clear that

$$
A_{*}(S) \leqq U(\Im ;[\Phi, \Im])=e A(S)
$$

In view of the relations established in I, 7 and IV, 14, the lemma is now proved.

The reader will notice that attention is restricted here to surfaces having a representation of non-parametric origin where the parameter range is an interval. Further considerations would establish this result more generally, but these are not necessary since it has been customary in the literature on such surfaces to so restrict the range of the independent variables.

16. The lemmas in III, 7,$8 ;$ IV , 4, 10, 11, 15 imply all the results of

( $\left.{ }^{9}\right)$ These are the expressions of Geöcze (see Rado [1]). 
Geöcze and Tonelli for the non-parametric case which are analogous to those for curves stated in 1 . For if $S$ be a surface having a representation $[x(u), \Im]$ of non-parametric origin

1.

$$
x(u)=\left(u^{1}, u^{2}, x^{3}\left(u^{1}, u^{2}\right)\right), \quad u=\left(u^{1}, u^{2}\right) \text { in the interval } \Im,
$$

then $e A(S)=A(S)$ by the lemma in IV, 15. Thus from IV, 4 it follows that a necessary and sufficient condition that the Lebesgue area of $S$ be finite is that each of the projection surfaces ${ }^{1} S,{ }^{2} S,{ }^{3} S$ be BEV. The projection surface ${ }^{3} S$ is obviously $\mathrm{BEV}$ under any conditions, while it follows from the lemma in III, 8 that a necessary and sufficient condition that ${ }^{1} S$ and ${ }^{2} S$ be BEV is that $x^{3}(u)$ be BV $T$ on $\Im$. Summarizing these facts, one obtains a known theorem in the non-parametric case (see Tonelli [1]): a necessary and sufficient condition that the Lebesgue area $A(S)$ of a surface $S$ having a representation 1 of non-parametric origin be finite is that $x^{3}(u)$ be BV $T$ on $\Im$.

If $x^{3}(u)$ is BV $T$ on $\Im$, it follows that the triple $J(u,[x, \Im])$ of ordinary Jacobians exists almost everywhere on $\Im$-in fact,

$$
J(u,[x, \Im])=\left(-x_{u^{1}}^{3}(u), \dot{x}_{u^{2}}^{3}(u), 1\right) \text { almost everywhere on } \Im .
$$

Hence from IV, 10 one concludes that this triple is summable on $\Im$ and

$$
A(S) \geqq \int_{\Im_{0}}\|J(u,[x, \Im])\| d u=\int_{\Im}\left[x_{u^{1}}^{3}(u)^{2}+x_{u^{2}}^{3}(u)^{2}+1\right]^{1 / 2} d u .
$$

In view of the lemma in III, 7, it is clear that the representation $[x(u), \Im]$ is BV $\Im^{0}$, hence BV $E$, when $x^{3}(u)$ is BV $T$ on $\Im$; from IV, 11 it follows that a necessary and sufficient condition that the sign of equality hold above is that $[x(u), \Im]$ be AC $E$. The representation $\left[{ }^{3} x(u), \Im\right]$ for ${ }^{3} S$ is clearly AC $E$ under any conditions, while it follows from the lemma in III, 8 that a necessary and sufficient condition that the representations $\left[{ }^{1} x(u), \Im\right],\left[{ }^{2} x(u), \Im\right]$ be AC $E$ is that $x^{3}(u)$ be AC $T$ on $\Im$. Thus other known theorems for the nonparametric case are obtained (see Tonelli [1]): if the Lebesgue area $A(S)$ is finite, then each of the partial derivatives

$$
x_{u^{1}}^{3}(u), x_{u^{2}}^{3}(u)
$$

exists almost everywhere in $\Im$, is summable on $\Im$ and

$$
A(S) \geqq \int_{\Im}\left[x_{u^{1}}^{3}(u)^{2}+\stackrel{3}{x_{u^{2}}(u)^{2}+1}\right]^{1 / 2} d u ;
$$

a necessary and sufficient condition that the sign of equality hold here is that $x^{3}(u)$ be AC $T$ on $\Im$.

17. The next sections are devoted to a comparison of the essential area defined in IV, 3 with the Geöcze area as defined by Rado (see Rado [2, part II, $\S 1])$. Given a continuous surface $S$, let $[x(u), \mathfrak{B}]$ be any representation for it. Define (see II, 2, 22), for any simple Jordan region $B$ in $\mathfrak{B}$, 


$$
\begin{aligned}
& { }^{i} \psi(B)=\left|\left(\bar{\Omega} 1,{ }^{i} T, B\right)\right|=\left|{ }^{i} T\left(E\left({ }^{i} T, B^{0}\right)\right)\right|=\int c\left({ }^{i} x,{ }^{i} T, B^{0}\right) d^{i} x, \quad i=1,2,3 ; \\
& \psi(B)=\left({ }^{1} \psi(B),{ }^{2} \psi(B),{ }^{3} \psi(B)\right), \quad \Psi(B)=\|\psi(B)\| .
\end{aligned}
$$

The quantity $U(\mathfrak{B} ;[\Psi, \mathfrak{B}])$ is independent of the choice of a representation for $S$ (see Rado [2]). It is this quantity which Rado terms the Geöcze area of $S$-denote it by $\mathcal{G}(S)$. Since $\AA\left(1,{ }^{i} T, B\right)$ is the set of points where $\mathrm{K}\left({ }^{i} x,{ }^{i} T\right.$, $\left.B^{0}\right)>0$, it follows at once that (see IV, 2,3$) \psi(B) \leqq \phi(B)$, hence $\Psi(B) \leqq \Phi(B)$ for $B$ in $\mathfrak{B}$, and $U(\mathfrak{B},[\Psi, \mathfrak{B}]) \leqq U(\mathfrak{B},[\Phi, \mathfrak{B}])$. Therefore one has the

Leмma. The Geöcze area does not exceed the essential area-that is, $G(S)$ $\leqq e A(S)$.

18. Retain the notation of the preceding section. Notice that (see II, $\mathbf{3}, \mathbf{6}$, 7) for any closed oriented square $s$ in $\mathfrak{B}$, it is so that

$$
\Psi(s) \geqq i(s) \geqq\left|{ }^{i} T\left(s^{0} \cdot \mathcal{E}\left({ }^{i} T, \mathfrak{B}^{0}\right)\right)\right|=G\left(s,{ }^{i} T, \mathcal{E}\left({ }^{i} T, \mathfrak{B}^{0}\right)\right), \quad i=1,2,3 .
$$

Thus a necessary condition that the Geöcze area $G(S)$ be finite is that each of the representations $\left[{ }^{i} x(u), \mathfrak{B}\right]$ for the projection surfaces ${ }^{i} S$ be BV $\mathcal{E}$ for $i=1,2,3$. This result implies the

LEMMA. If a surface $S$ possesses a representation $[x(u), \mathfrak{B}]$ which is not $\mathrm{BV} \mathcal{E}$-that is, for which at least one of the representations $\left[{ }^{i} x(u), \mathfrak{B}\right]$ is not $\mathrm{BV} \mathcal{E}$-then $G(S)=e A(S)=+\infty$.

19. LEMMA. If a surface $S$ possesses a representation $[x(u), \mathfrak{B}]$ which is BV $E$, then $G(S)=e A(S)<+\infty$.

Proof. From III, 3 ; IV, 4 it follows that $e A(S)$ is finite. In view of the lemma in IV, 17 it is sufficient to show that $G(S) \geqq e A(S)$. Given a positive number $\epsilon$, there exists a finite system $S(\mathfrak{B})$ such that $\Phi(S(\mathfrak{B}))>e A(S)-\epsilon$. Since $[x(u), \mathfrak{B}]$ is $\mathrm{BV} E$, one concludes from the lemma in II, 20 (see IV, 1) that there exists a sequence of subdivisions $S_{n}(B)$ for each $B$ in $\mathfrak{B}$ such that $\left\|S_{n}(B)\right\|$ is less than $n^{-1}$ and each $S_{n}(B)$ is maximal for each of the transformations $\left[{ }^{i} x_{n}(u), B\right]$ for $i=1,2,3$. From the lemma in II, 22 (see IV, 2, 17), it follows that ${ }^{i} \phi(B)=\lim i \psi\left(S_{n}(B)\right)$ for $i=1,2,3 ; B$ in $S(\mathfrak{B})$. Denote by $S_{n}(\mathfrak{B})$ the finite system consisting of all simple Jordan regions belonging to an $S_{n}(B)$ for some $B$ in $S(\mathfrak{B})$. From the triangle inequality one finds that

$$
G(S) \geqq \Psi\left(S_{n}(\mathfrak{B})\right) \geqq \sum_{B}\left[\sum_{i=1}^{3} \psi(\mathfrak{B})\left(S_{n}(B)\right)^{2}\right]^{1 / 2} \rightarrow_{n \rightarrow \infty} \Phi(S(\mathfrak{B}))>e A(S)-\epsilon_{.}
$$

Thus $G(S)>e A(S)-\epsilon$, and since $\epsilon$ is arbitrary, the lemma follows.

20. Summarizing the lemmas in IV, 18, 19, one concludes that the essential area and the Geöcze area of a surface are equal if the surface either has a representation which is not $\mathrm{BV} \mathcal{E}$, or has a representation which is $\mathrm{BV} E$. 
This leaves open the question of whether these areas are equal if all the representations of a surface are $\mathrm{BV} \mathcal{E}$, but none is $\mathrm{BV} E$. Indeed, a first question might be whether such surfaces exist. A negative answer to this question would enable one to close the gap between necessary conditions and sufficient conditions in the results in IV, 6, 10.

21. For applications in the next chapter, the following result will be useful. Assume that a surface $S$ has a representation which is $\mathrm{BV} E$; in view of the lemma in II, 18, one may assume that this representation has the form $[x(u), \Im]$ where $\Im$ is an interval. Now the essential area $e A(S)$ is finite (see IV , 4). It follows from the lemma in II, 20 that there exist interval subdivisions $S_{n}(\Im)$ such that $\left\|S_{n}(\Im)\right\|$ converges to zero and each $S_{n}(\Im)$ is maximal for each of the transformations $\left[{ }^{i} x(u), \Im\right]$ for $i=1,2,3$; for brevity, $S_{n}(\Im)$ is said to be maximal for $[x(u), \Im]$. Given a positive number $\epsilon$, let $S(\Im)$ be a finite system of nonoverlapping Jordan regions such that $\Phi(S(\Im))>e A(S)-\epsilon$. For each $B$ in $S(\Im)$, denote by $u(B)$ an arbitrary point in the interior of $B$. For each integer $n$ and each $B$ in $S(\Im)$, denote by $S_{n}(B)$ the maximal collection of those intervals in $S_{n}(\Im)$ whose point set sum $\beta_{n}$ is a simple Jordan region containing $u(B)$ and lying in the interior of $B$. It follows (see Kerékjártó [1]) that the $\beta_{n}$ fill up $B$ from the interior (see 4). Consequently one obtains (see II, 2, 2; IV, 2)

$$
\begin{array}{cc}
{ }^{i} \phi\left(S_{n}(B)\right)={ }^{i} \phi\left(\beta_{n}\right) \rightarrow_{n \rightarrow \infty}{ }^{i} \phi(B) & \text { for } i=1,2,3 ; B \text { in } S(\Im), \\
e A(S) \geqq \Phi\left(S_{n}(\Im)\right) \geqq \sum_{B \text { in } S(\Im)} \Phi\left(S_{n}(B)\right) \geqq \sum_{B \text { in } S(\Im)} \Phi\left(\beta_{n}\right) \rightarrow_{n \rightarrow \infty} \Phi(S(\Im)) \\
>e A(S)-\epsilon .
\end{array}
$$

In view of $\mathrm{IV}, 3$, this implies the

LEMMA. Let $S$ be a surface having a representation which is $\mathrm{BV} E$. Then there are representations $[x(u), \Im]$ for $S$ which are $\mathrm{BV} E$, and for every sequence of interval subdivisions $S_{n}(\Im)$ such that $\left\|S_{n}(\Im)\right\|$ converges to zero and each $S_{n}(\Im)$ is maximal with respect to $[x(u), \mathfrak{B}]$, it is true that $\lim \Phi\left(S_{n}(\Im)\right)=e A(S)$.

\section{Chapter V}

\section{Applications}

1. In a study of convergence in area, Rado and Reichelderfer (see $\mathrm{R}^{2}$ [2]) obtained the following results. Let $S_{n}, n=0,1,2, \cdots$, be a sequence of continuous surfaces having representations $\left[x_{n}(u), \Im\right]$ of non-parametric origin (see I, 2), where

$$
x_{n}(u)=\left(u^{1}, u^{2}, x_{n}^{3}\left(u^{1}, u^{2}\right)\right) \text { for } u=\left(u^{1}, u^{2}\right) \text { in } \Im, \quad n=0,1,2, \cdots .
$$

Make the following assumptions: the functions $x_{n}^{3}(u)$ converge on $\Im$ uniformly to $x_{0}^{3}(u)$; each of the functions $x_{n}^{3}(u)$ for $n=0,1,2, \ldots$ is BV $T$ on $\Im$ 
(see III, 5); and the Lebesgue areas $A\left(S_{n}\right)$ converge to $A\left(S_{0}\right)$ (see I, 6). Then the total variations $V_{u^{1}}\left(x_{n}^{3}\right), V_{u^{2}}\left(x_{n}^{3}\right)$ converge to $V_{u^{1}}\left(x_{0}^{3}\right), V_{u^{2}}\left(x_{0}^{3}\right)$, respectively (see $\mathrm{R}^{2}$ [2, chap. I, §19]). Observe that the first assumption implies that $S_{n}$ converges to $S_{0}$ (see I, 1 ).

2. Suppose that $S$ is a continuous surface having a representation $[x(u), \Im]$ for which the triple of ordinary Jacobians $J(u ;[x, \Im])$ exists almost everywhere in $\Im$ (see IV, 1 ) and the Lebesgue area $A(S)$ is finite; then the triple $J\left(u,[x, \Im]\right.$ ) is summable on $\Im$ (see IV, 10, 14). Assume that $S_{n}, n=1$, $2, \cdots$, is a sequence of continuous surfaces having representations $\left[x_{n}(u), \Im\right]$ satisfying the following conditions: the functions $x_{n}^{i}(u)$ converge on $\Im$ uniformly to $x^{i}(u)$ for $i=1,2,3$; each of the representations $\left[x_{n}(u), \Im\right]$ for $n=1,2, \cdots$, is absolutely continuous $(A, J)$ (see $\mathrm{I}, 8)$; and the Lebesgue areas $A\left(S_{n}\right)$ converge to $\int_{\Im}\|J(u,[x, \Im])\| d u$. Then Rado and Reichelderfer (see $\mathrm{R}^{2}$ [2, chap. I, $\left.\$ 25-27\right]$ ) show that

$$
\lim \int_{\Im}\left|J\left(u,\left[{ }^{i} x_{n}, \Im\right]\right)\right| d u=\int_{\Im}\left|J\left(u,\left[{ }^{i} x, \Im\right]\right)\right| d u \quad \text { for } i=1,2,3 .
$$

Since sequences of surfaces $S_{n}$ having the properties just described exist if and only if the representation $[x(u), \Im]$ for $S$ is absolutely continuous $(A, J)$, they show that if $[x(u), \Im]$ is absolutely continuous $(A, J)$, then each of the representations $\left[{ }^{i} x(u), \Im\right]$ for the projection surfaces ${ }^{i} S$ for $i=1,2,3$ is also absolutely continuous $(A, J)$, and each of the transformations $\left[{ }^{i} x(u), \mathfrak{\Im}^{0}\right]$ for $i=1,2,3$ belongs to the class $K_{3}$ described in II, 8. Observe that the first condition on the $\left[x_{n}(u), \Im\right]$ implies that $S_{n}$ converges to $S_{0}$. These results of Rado and Reichelderfer will appear as corollaries to more general theorems whch are presented in the wake of certain preliminary notions (see $\mathrm{V}, \mathbf{8}, \mathbf{1 5}$ ).

3. Let $\Im$ be any interval in the $u$-plane. A class $\mathcal{F}$ of intervals $I$ in $\Im$ is termed closed if it possesses the following properties.

1. The interval $\Im$ is in $\mathcal{F}$.

2 If $I_{1}$ and $I_{2}$ are in $\mathcal{F}$, and if $I_{1} \cdot I_{2}$ is an interval, then $I_{1} \cdot I_{2}$ is in $\mathcal{F}$.

3. If $I$ is any interval in $\mathcal{F}$, then there exists an interval subdivision $S(\Im)$ in $\mathcal{F}$ which contains $I$ as an element.

A particular type of closed class is important for the sequel. Let $\mathcal{L}$ be a class containing an at most enumerable number of lines $\left({ }^{10}\right)$, each of which is parallel to one of the coordinate axes in the $u$-plane, and none of which forms a side of the interval $\Im$. Denote by $\mathfrak{F}(\mathcal{L})$ the class of all intervals $I$ in $\Im$, each of whose four sides is formed by a segment of a line not in $\mathcal{L}$. It is readily verified that $\mathcal{F}(\mathcal{L})$ is a closed class. If $\mathcal{L}_{1}, \mathcal{L}_{2}, \cdots$ be a finite or enumerably infinite set of classes of lines each of type $\mathcal{L}$, then the class $\mathcal{F}$ of all the intervals found in every one of the classes $\mathscr{F}\left(\mathcal{L}_{1}\right), \mathfrak{F}\left(\mathcal{L}_{2}\right), \ldots$ is again a closed class having the same structure as $\mathscr{F}(\mathcal{L})$. A closed class of type $\mathcal{F}(\mathcal{L})$ is termed $c$-closed.

${ }^{(10)}$ The class $\mathcal{L}$ may be empty. 
Let $\mathcal{F}$ be any closed class. Assume that to every interval $I$ in $\mathcal{F}$ there is associated a finite real number $\phi(I)$; this function is termed an interval function of $\mathcal{F}$, and denoted by $[\phi, \mathcal{F}]$. For every interval $I$ in $\mathcal{F}$, define (cf. 4)

$$
u(I ;[\phi, \mathcal{F}])=\text { l.u.b. } \phi(S(I)) \text { for finite interval systems } S(I) \text { in } \mathcal{F} \text {. }
$$

Evidently $\phi(I) \leqq u(I ;[\phi, \mathcal{F}]) \leqq u(\Im ;[\phi, F])$ for $I$ in $F$. A necessary and sufficient condition that $[u, \mathcal{F}]$ be an interval function on $\mathcal{F}$ is that $u(\mathcal{F} ;[\phi, \mathcal{F}])$ be finite; if $[u, \mathcal{F}]$ is an interval function, then $[\phi, \mathcal{F}]$ is said to possess a $u$-function.

4. Let $S$ be a continuous surface having a representation which is $\mathrm{BV} E$; as noted in IV, 21, one may without loss of generality assume this representation to be of the form $[x(u), \Im]$, where $\Im$ is an interval. Let $\mathcal{F}([x, \Im])$ denote the class of all intervals $I$ in $\Im$ having the following property: for each of the four lines $l$, a segment of which forms the boundary of $I$, it is true that (cf. IV, 1) $\left|{ }^{i} T\left(l \cdot E\left({ }^{i} T, \Im^{0}\right)\right)\right|=0$ for $i=1,2,3$ where ${ }^{i} T$ is the transformation $\left[{ }^{i} x(u), \Im^{0}\right]$. From the corollary in II, 17 , it is clear that $\mathcal{F}([x, \Im])$ is a $c$-closed class. Let $I$ be any interval in $\mathcal{F}([x, \Im])$ and let $S(I)$ be any interval subdivision in $\mathfrak{F}([x, \Im])$. Then (see II, $19 ; \mathrm{IV}, 2,21) S(I)$ is a maximal system for $[x(u), \Im]$, so that

1.

$$
{ }^{i} \phi(S(I))={ }^{i} \phi(I) \quad \text { for } \quad i=1,2,3 ; \quad \Phi(S(I)) \geqq \Phi(I) .
$$

Let $\mathcal{F}$ be any $c$-closed subset of $\mathscr{F}([x, \Im])$. Then for every interval $I$ in $\mathcal{F}$, there necessarily exists a sequence of interval subdivisions $S_{n}(I)$ in $\mathcal{F}$ such that $\left\|S_{n}(I)\right\|$ converges to zero. From IV, 2, 21, one finds (see 4; V, 3), for $I$ in $\mathcal{F}$,

2.

$$
\begin{aligned}
& U\left(I ;\left[{ }^{i} \phi, \Im\right]\right)=u\left(I ;\left[{ }^{i} \phi, \mathcal{F}\right]\right)={ }^{i} \phi(I) \quad \text { for } i=1,2,3 ; \\
& U(I ;[\Phi, \Im])=u(I ;[\Phi, \mathcal{F}]) .
\end{aligned}
$$

5. General Lemma. Let $\left[\phi_{n}, \mathcal{F}\right]$ where $\phi_{n}(I)=\left({ }^{1} \phi_{n}(I),{ }^{2} \phi_{n}(I),{ }^{3} \phi_{n}(I)\right)$ for I in $\mathcal{F}, n=0,1,2, \cdots$, be a sequence of triples of interval functions defined on a closed class $F$. Set $\Phi_{n}(I)=\left\|\phi_{n}(I)\right\|$ for I in $\mathcal{F}$. Make the following assumptions.

1. Each $\left[{ }^{i} \phi_{n}, \mathcal{F}\right]$ is non-negative for $i=1,2,3 ; n=0,1,2, \cdots$.

2. Each $\left[{ }^{i} \phi_{n}, \mathcal{F}\right]$ has a u-function for $i=1,2,3 ; n=0,1,2, \cdots$.

3. If $I$ be any interval in $F$, if $S(I)$ be any interval subdivision in $F$, then ${ }^{i} \phi_{n}(S(I)) \geqq{ }^{i} \phi_{n}(I)$ for $i=1,2,3 ; n=0,1,2, \cdots$.

4. $\lim \inf { }^{i} \phi_{n}(I) \geqq{ }^{i} \phi_{0}(I)$ for $I$ in $\mathcal{F} ; i=1,2,3$.

5. $\lim u\left(\Im ;\left[\Phi_{n}, \mathcal{F}\right]\right)=u\left(\Im ;\left[\Phi_{0}, \mathcal{F}\right]\right)$.

Then $\lim u\left(I,\left[{ }^{i} \phi_{n}, \mathcal{F}\right]\right)=u\left(I ;\left[{ }^{i} \phi_{0}, \mathcal{F}\right]\right)$ for $I$ in $\mathcal{F}, i=1,2,3$.

For the special case when $\mathcal{F}$ consists of all the intervals in $\Im$, this lemma is stated and proved by Rado and Reichelderfer (see $\mathrm{R}^{2}$ [2, chap. II, $\left.\S \S 1-8\right]$ ). A proof for this slightly more general lemma may be made by using the properties of a closed class (see V, 3), and following step by step their proof. 
6. A central result in this chapter may now be stated and proved.

ThEOREM. Let $S_{n}, n=0,1,2, \cdots$, be a sequence of continuous surfaces satisfying the following conditions.

1. The surfaces $S_{n}$ converge to $S_{0}$ (see I, 1).

2. Each of the surfaces $S_{n}$ for $n=0,1,2, \cdots$ has a representation which is BV $E$ (see IV, 1).

3. The essential areas $e A\left(S_{n}\right)$ converge to eA $\left(S_{0}\right)$.

Then the essential variations $\mathrm{eV}\left({ }^{i} S_{n}\right)$ for the projection surfaces ${ }^{i} S_{n}$ converge to $\mathrm{e}\left({ }^{i} S_{0}\right)$ for $i=1,2,3$.

Proof. From conditions 1, 2; IV, 21; I, 1, it follows that there exist representations $\left[x_{n}(u), \Im\right]$ for $S_{n}$ each having the same in terval of definition $\Im$, and each BV $E$, such that $x_{n}(u)$ converges uniformly on $\Im$ to $x_{0}(u)$. Let $\mathfrak{F}$ denote the class of all intervals belonging to every one of the $c$-closed classes, $\mathcal{F}\left(\left[x_{n}, \Im\right]\right)$ for $n=0,1,2, \ldots$ (see $\left.\mathrm{V}, 4\right)$; then $\mathcal{F}$ is a $c$-closed class. Define triples of $b$-functions $\left[\phi_{n}, \Im\right]$ as in IV, 2 for $n=0,1,2, \ldots$ From V, 4, 2, one obtains, for $I$ in $\mathcal{F}$ (see III, $3 ;$ IV, 3), $n=0,1,2, \cdots$,

4.

$$
\begin{aligned}
& U\left(I ;\left[{ }^{i} \phi_{n}, \Im\right]\right)=u\left(I ;\left[{ }^{i} \phi_{n}, \mathcal{F}\right]\right)={ }^{i} \phi_{n}(I) \quad \text { for } i=1,2,3 \text {; } \\
& U\left(I ;\left[\Phi_{n}, \Im\right]\right)=u\left(I ;\left[\Phi_{n}, \mathcal{F}\right]\right) \text {; } \\
& e V\left({ }^{i} S_{n}\right)=u\left(\Im ;\left[{ }^{i} \phi_{n}, \mathcal{F}\right]\right)={ }^{i} \phi_{n}(\Im) \quad \text { for } i=1,2,3 \text {; } \\
& e A\left(S_{n}\right)=u\left(\Im,\left[\Phi_{n}, \Im\right]\right) \text {. }
\end{aligned}
$$

Thus conditions V, 5, 1, 2 are satisfied. From V, 4, 1, it is clear that V, 5, 3 is fulfilled. Condition V, 5, 4 follows at once from II, 2, 2 and the lemma of Fatou, since $x_{n}(u)$ converges on $\Im$ uniformly to $x_{0}(u)$. Finally, from relation 4 and condition 3 follows condition $V, 5,5$. The conclusion of this theorem thus follows at once from the lemma in V, 5 and relation 4.

7. COROLlARY. Let $S$ be a continuous, surface having a representation which is $\mathrm{BV} E$. If the Lebesgue area $A(S)$ equals the essential area $e A(S)$, then the Lebesgue areas $A\left({ }^{i} S\right)$ of the projection surfaces ${ }^{i} S$ equal the essential areas $e A\left({ }^{i} S\right)$ for $i=1,2,3$.

Proof. Let $P_{n}, n=1,2, \cdots$, be a sequence of polyhedra such that $P_{n}$ converges to $S$ and $A\left(P_{n}\right)$ converges to $A(S)$ (see I, 6,2). Since $A\left(P_{n}\right)=e A\left(P_{n}\right)$ and each $P_{n}$ has a representation which is AC $E$ (see IV, 14) for $n=1,2 \cdots$, it follows that the hypotheses of the theorem in V, 6 are fulfilled by $P_{n}$ and $S$. Thus $e V\left({ }^{i} P_{n}\right)$ converges to $e V\left({ }^{i} S\right)$ for $i=1,2,3$. But since ${ }^{i} P_{n}$ is a flat polyhedron, it is true that $e V\left({ }^{i} P_{n}\right)=e A\left({ }^{i} P_{n}\right)=A\left({ }^{i} P_{n}\right)$ for $i=1,2,3 ; n=1,2, \cdots$ (see IV, 3, 14); also $e V\left({ }^{i} S\right)=e A\left({ }^{i} S\right)$ for $i=1,2$, 3. Since $i P_{n}$ converges to $i S$, it follows that (see I, 6, 3)

$$
A\left({ }^{i} S\right) \leqq \lim \inf A\left({ }^{i} P_{n}\right)=\lim \inf e V\left({ }^{i} P_{n}\right)=e A\left({ }^{i} S\right) \quad \text { for } i=1,2,3 .
$$

The conclusion of the corollary follows from this inequality and IV, 14. 
8. Let $S_{n}, n=0,1,2, \cdots$, be a sequence of continuous surfaces each possessing a representation $\left[x_{n}(u), \Im_{n}\right]$ of non-parametric origin (see I, 2), where

1. $x_{n}(u)=\left(u^{1}, u^{2}, x_{n}^{3}\left(u^{1}, u^{2}\right)\right)$ for $u=\left(u^{1}, u^{2}\right)$ in $\Im_{n}, \quad n=0,1,2, \ldots$.

Then (see IV, 15) the essential area $e A\left(S_{n}\right)$ equals the Lebesgue area $A\left(S_{n}\right)$ for $n=0,1,2, \cdots$. Suppose that $S_{n}$ converges to $S_{0}$; it follows then that $\Im_{n}$ converges to $\Im_{0}$, and $x_{n}^{3}(u)$ converges uniformly on every closed set in the interior of $\Im_{0}$ to $x_{0}^{3}(u)$. Now assume that each of the Lebesgue areas $A\left(S_{n}\right)$ is finite for $n=0,1,2, \ldots$; this implies (see II, 7; IV, 16) that each of the representations $\left[x_{n}(u), \Im_{n}\right]$ is BV $E$. Thus to the theorem in V, 6 there follows the

Corollary. Let $S_{n}, n=0,1,2, \cdots$, be a sequence of continuous surfaces, each possessing a representation 1 of non-parametric origin and a finite Lebesgue area $A\left(S_{n}\right)$. If the surfaces $S_{n}$ converge to $S_{0}$, if the areas $A\left(S_{n}\right)$ converge to $A\left(S_{0}\right)$, then the variations e $V\left({ }^{i} S_{n}\right)$ for the projection surfaces ${ }^{i} S_{n}$ converge to e $V\left({ }^{i} S_{0}\right)$ for $i=1,2,3$.

In view of the lemma in III,8 (see IV,16), this result is clearly a generalization of that of Rado and Reichelderfer cited in V, 1.

9. A second important result in this chapter is contained in the

TheOREM. Let $S_{n}, n=0,1,2, \cdots$, be a sequence of continuous surfaces satisfying the following conditions:

1. the surfaces $S_{n}$ converge to $S_{0}$ (see I, 1);

2. the surface $S_{0}$ has a representation $\left[x_{0}(u), \mathfrak{B}_{0}\right]$ for which the triple $\mathcal{F}\left(u,\left[x_{0}, \mathfrak{B}_{0}\right]\right)$ of generalized Jacobians exists almost everywhere in $\mathfrak{B}_{0}^{0}$, is summable on $\mathfrak{B}_{0}^{0}($ see IV, 1$)$;

3. the surfaces $S_{n}$ for $n=1,2, \cdots$ have representations which are $\mathrm{AC} E$;

4. the essential areas $e A\left(S_{n}\right)$ converge to $\int_{\mathfrak{O}_{0}^{\circ}}\left\|\mathcal{F}\left(u,\left[x_{0}, \mathfrak{B}_{0}\right]\right)\right\| d u$.

Then

5. the representation $\left[x_{0}(u), \mathfrak{B}_{0}\right]$ is $\mathrm{AC} E$;

6. the representation $\left[x_{0}(u), \mathfrak{B}_{0}\right]$ is absolutely continuous $(e A, \mathcal{F})($ see $\mathrm{I}, 8)$;

7. the essential variations $\mathrm{e} V\left({ }^{i} S_{n}\right)$ for the projection surfaces ${ }^{i} S_{n}$ converge to $e V\left({ }^{i} S_{0}\right)$ for $i=1,2,3$.

In view of the theorem in IV, 9, it is clear that conclusion 5 implies conclusion 6 ; if conclusions 5, 6 are true, then the hypotheses of the theorem in $\mathrm{V}, 6$ are fulfilled, so conclusion 7 follows. It suffices therefore to prove 5 . This proof is divided into two parts: an "assume without loss of generality" section $(\mathrm{V}, 10)$, and the proof itself $(\mathrm{V}, 11)$.

10. No loss of generality is imposed in the preceding theorem if the following additional assumptions are made:

1. the simple Jordan regions $\mathfrak{B}_{n}$ fill up $\mathfrak{B}_{0}$ from the interior (see 4 ); 
2. on every closed set in the interior of $\mathfrak{B}_{0}, x_{n}(u)$ converges uniformly to $x_{0}(u)$.

In proving this, a theorem of Franklin and Wiener is useful (see Franklin and Wiener [1]). Given a topological map $[\bar{u}(u), \mathfrak{B}]$ of a Jordan region $\mathfrak{B}$ in the $u$-plane onto a Jordan region $\bar{B}$ and a positive constant $\epsilon$, there exists a pair of analytic functions $\left[\bar{u}_{\epsilon}(u), \Re\right]$ defining a topological map of some Jordan region $\Re$ in the $u$-plane containing $\mathfrak{B}$ in its interior on to a Jordan region $\bar{\Re}$ containing $\overline{\mathfrak{B}}$ in its interior, and such that $\left\|\bar{u}_{\epsilon}(u)-\bar{u}(u)\right\|<\epsilon$ for $u$ in $\mathfrak{B}$ and $\left\|\bar{u}_{\epsilon}^{-1}(u)-\bar{u}^{-1}(u)\right\|<\epsilon$ for $u$ in $\overline{\mathfrak{B}}$, where $\left[\bar{u}^{-1}(u), \overline{\mathfrak{B}}\right],\left[\bar{u}_{\epsilon}^{-1}(u), \bar{\Re}\right]$ are the inverse maps of $[\bar{u}(u), \mathfrak{B}],\left[\bar{u}_{\epsilon}(u), \Re\right]$, respectively. Let $\mathfrak{B}_{\epsilon}$ denote the correspond to $\overline{\mathfrak{B}}$ under the map $\left[\bar{u}_{\epsilon}^{-1}(u), \overline{\mathfrak{R}}\right]$. Then $\left[\bar{u}^{-1}\left(\bar{u}_{\epsilon}(u)\right), \mathfrak{B}_{\epsilon}\right]$ is a topological map of $\mathfrak{B}_{\epsilon}$ onto $\mathfrak{B}$ such that $\left\|\bar{u}^{-1}\left(\bar{u}_{\epsilon}(u)\right)-u\right\|<\epsilon$ for $u$ in $\mathfrak{B}_{\epsilon}$. Suppose that $S$ is a surface having a representation $[\bar{x}(u), \overline{\mathfrak{B}}]$ which is AC $E$; consider the representation $\left[\bar{x}\left(\bar{u}_{\epsilon}(u)\right), \mathfrak{B}_{\epsilon}\right]$ for $S$. Denote by $M$ the maximum of the absolute value of the ordinary Jacobian $J\left(u,\left[\bar{u}_{\epsilon}, \mathfrak{B}_{\epsilon}\right]\right)$. Then a simple Jordan region $\bar{B}$ in $\overline{\mathfrak{B}}$ which is the image of a square $s$ in $\mathfrak{B}_{\epsilon}$ under $\left[\bar{u}_{\epsilon}(u), \mathfrak{B}_{\epsilon}\right]$ has an area not exceeding $M \cdot|s|$. Let ${ }^{i} \bar{T},{ }^{i} T$ denote transformations $\left[{ }^{i} \bar{x}(u), \overline{\mathfrak{B}}\right],\left[{ }^{i} \bar{x}\left(\bar{u}_{\epsilon}(u)\right), \mathfrak{B}_{\epsilon}\right]$ respectively, for $i=1,2,3$. Then (see II, 1-9), since $[\bar{x}(u), \overline{\mathfrak{B}}]$ is AC $E$,

$$
\begin{aligned}
G\left(s,{ }^{i} T, E\left({ }^{i} T, \mathfrak{B}_{\epsilon}^{0}\right)\right) & =\mid{ }^{i} T\left(s ^ { 0 } \cdot E ( { } ^ { i } T , \mathfrak { B } _ { \epsilon } ^ { 0 } ) | = | { } ^ { i } \overline { T } \left(\bar{B}^{0} \cdot E\left({ }^{i} \bar{T}, \overline{\mathfrak{B}}^{0}\right) \mid\right.\right. \\
& \leqq \int \mathrm{K}\left({ }^{i} x,{ }^{i} \bar{T}, \bar{B}^{0}\right) d^{i} x=\int_{\bar{B}^{0}} D\left(u,{ }^{i} \bar{T}\right) d u \quad \text { for } i=1,2,3 .
\end{aligned}
$$

Thus $\left[\bar{x}\left(\bar{u}_{\epsilon}(u)\right), \mathfrak{B}_{\epsilon}\right]$ is also AC $E$. Now the representation $[\bar{x}(\bar{u}(u)), \mathfrak{B}]$ need not be AC $E$. And if $[x(u), \mathfrak{B}]$ is an arbitrary preassigned representation for $S$, and $\zeta$ is any positive number, then $[\bar{u}(u), \mathfrak{B}]$ may be so chosen that $\|\bar{x}(\bar{u}(u))-x(u)\|<\zeta$ for $u$ in $\mathfrak{B}$ (see $\mathrm{I}, 1)$. These results are summarized in the

LEMMA. Let $S$ be a continuous surface possessing a representation which is $\mathrm{AC} E$. If $[x(u), \mathfrak{B}]$ is an arbitrary representation for $S$, and if $\epsilon$ and $\zeta$ are any positive numbers, then there exists a simple Jordan region $\mathfrak{B}_{\epsilon}$, a topological map $\left[u_{\epsilon}(u), \mathfrak{B}_{\epsilon}\right]$ of $\mathfrak{B}_{\epsilon}$ onto $\mathfrak{B}$ such that $\left\|u_{\epsilon}(u)-u\right\|<\epsilon$ for $u$ in $\mathfrak{B}_{\epsilon}$, and an AC $E$ representation $\left[x_{\epsilon}(u), \mathfrak{B}_{\epsilon}\right]$ such that $\left\|x_{\epsilon}(u)-x\left(u_{\epsilon}(u)\right)\right\|<\zeta$ for $u$ in $\mathfrak{B}_{\epsilon}$.

Choose positive numbers $\epsilon_{n}$ such that $\left\|x_{0}\left(u^{\prime}\right)-x_{0}\left(u^{\prime \prime}\right)\right\|<n^{-1}$ for any points $u^{\prime}, u^{\prime \prime}$ in $\mathfrak{B}_{0}$ satisfying $\left\|u^{\prime}-u^{\prime \prime}\right\|<\epsilon_{n}$. Let $B_{n}$ be a Jordan region in the interior of $\mathfrak{B}_{0}$ for which there exists a topological map $\left[u_{n}(u), B_{n}\right]$ of $B_{n}$ onto $\mathfrak{B}_{0}$ such that $\left\|u_{n}(u)-u\right\|<\epsilon_{n}$ for $u$ in $B_{n}, n=1,2, \ldots$. Then $\left\|x_{0}\left(u_{n}(u)\right)-x_{0}(u)\right\|$ $<n^{-1}$. Thus the simple Jordan regions $B_{n}$ fill up $\mathfrak{B}_{0}$ from the interior (see 4 ), the surfaces $S_{0 n}$ having representations $\left[x_{0}(u), B_{n}\right]$ satisfy $d\left(S_{0}, S_{0 n}\right)<n^{-1}$, and since clearly $e A\left(S_{0 n}\right) \leqq e A\left(S_{0}\right)$, it follows that $e A\left(S_{0_{n}}\right)$ converges to $e A\left(S_{0}\right)$ (see IV, 5). Now $d\left(S_{n}, S_{0 n}\right)<d\left(S_{n}, S_{0}\right)+n^{-1}$ and so the surfaces $S_{n}$ admit representations $\left[\sharp x_{n}(u), B_{n}\right]$ for which $\left\|\sharp x_{n}(u)-x_{0}(u)\right\|<d\left(S_{n}, S_{0}\right)+n^{-1}$ for $u$ in $B_{n}, n=1,2, \cdots$ (see I, 1$)$. Since the surfaces $S_{n}$ have representations 
which are AC $E$ for $n=1,2, \cdots$ (see $\mathrm{V}, 9,3$ ), and since $B_{n}$ is in the interior of $\mathfrak{B}_{0}$, it follows by the preceding lemma that there exist simple Jordan regions ${ }_{*} \mathfrak{B}_{n}$ in $\mathfrak{B}_{0}$, topological maps $\left[{ }_{*} u_{n}(u),{ }_{*} \mathfrak{B}_{n}\right]$ of $* \mathfrak{B}_{n}$ onto $B_{n}$ such that $\|_{*} u_{n}(u)$ $-u \|<\epsilon_{n}$ for $u$ in $* \mathfrak{B}_{n}$ and AC $E$ representations $\left[* x_{n}(u), * \mathfrak{B}_{n}\right]$ such that $\left\|_{*} x_{n}(u)-* x_{n}\left(* u_{n}(u)\right)\right\|<n^{-1}$ for $u$ in $* \mathfrak{B}_{n}$. Since $\left\|x_{0}\left(* u_{n}(u)\right)-x_{0}(u)\right\|<n^{-1}$ for $u$ in $* \mathfrak{B}_{n}$, it follows that $\left\|_{*} x_{n}(u)-x_{0}(u)\right\|<d\left(S_{n}, S_{0}\right)+3 n^{-1}$ for $u$ in $* \mathfrak{B}_{n}$. The representations $\left[* x_{n}(u), * \mathfrak{B}_{n}\right]$ thus satisfy the hypotheses of the theorem in $\mathrm{V}, 9$ and the additional assumptions in this section.

11. A proof for the theorem in V, 9 is now made, using the additional conditions, V, 10, 1, 2. First, observe that V, 9 imply (see IV, 5, 6) that $e V\left(S_{0}\right)$ is finite, and

$$
\lim e A\left(S_{n}\right)=e A\left(S_{0}\right)=\int_{\mathfrak{B}_{0}^{0}}\left\|\mathcal{F}\left(u,\left[x_{0}, \mathfrak{B}_{0}\right]\right)\right\| d u .
$$

This verifies $\mathrm{V}, 9,6$ directly (see $I, 8)$. Let $I$ be any interval in the interior of $\mathfrak{B}_{0}$; in view of $\mathrm{V}, 10,1$, there exists an $n(I)$ such that $I$ is in the interior of $\mathfrak{B}_{n}$ for $n>n(I)$. Define (see IV, 1 ), for $I$ in $\mathfrak{B}_{0}^{0}$.

$$
\begin{array}{lrl}
i \psi_{n}(I)=\int_{I}\left|\mathcal{F}\left(u,{ }^{i} T_{n}\right)\right| d u & \text { for } i=1,2,3 ; \\
\psi_{n}(I)=\left({ }^{1} \psi(I),{ }^{2} \psi(I),{ }^{3} \psi(I)\right), & \Psi_{n}(I)=\left\|\psi_{n}(I)\right\| & \text { for } n=0, n>n(I) .
\end{array}
$$

From V, 9, 3, it is seen that (see II, 9; 1, IV, 3)

$$
{ }^{i} \psi_{n}(I)=\int \mathrm{K}\left({ }^{i} x,{ }^{i} T_{n}, I^{0}\right) d^{i} x \text { for } i=1,2,3, n>n(I) .
$$

Since $e A\left(S_{0}\right)$ is finite, it follows (see IV, 4) that $\mathrm{K}\left({ }^{i} x,{ }^{i} T_{0}, I^{0}\right)$ is summable, and (see V, 10, 2; II, 2, 2; II, 9, 1)

$$
\lim \inf \int \mathrm{K}\left({ }^{i} x,{ }^{i} T_{n}, I^{0}\right) d^{i} x \geqq \int \mathrm{K}\left({ }^{i} x,{ }^{i} T_{0}, I^{0}\right) d^{i} x \geqq{ }^{i} \psi_{0}(I) \quad \text { for } i=1,2,3 .
$$

These relations give

$$
\lim \inf ^{i} \psi_{n}(I) \geqq{ }^{i} \psi_{0}(I) \quad \text { for } i=1,2,3, I \text { in } \mathfrak{B}_{0}^{0} .
$$

By a known result (see $\mathrm{R}^{2}[2$, chap. II, $\S 10]$ ), it follows that for $I$ in $\mathfrak{B}_{0}^{0}$,

$$
u\left(I ;\left[\psi_{n}, I\right]\right)=i \psi_{n}(I)
$$

for $i=1,2,3$,

2.

$$
u\left(I ;\left[\Psi_{n}, I\right]\right)=\int_{I}\left\|\mathcal{F}\left(u,\left[x_{n}, \mathfrak{B}_{n}\right]\right)\right\| d u \quad \text { for } n=0, n>n(I) .
$$

A direct reasoning using relation 1 shows that

$$
\lim \inf u\left(I ;\left[\Psi_{n}, I\right]\right) \geqq u\left(I ;\left[\Psi_{0}, I\right]\right)
$$


For $n=0, n>n(I)$, the interval $I$ lies in the interior of $\mathfrak{B}_{0}$; extend the sides of $I$ until they meet the boundary of $\mathfrak{B}_{n}$ thus dividing $\mathfrak{B}_{n}$ into nine simple Jordan regions $I \equiv{ }_{0} B_{n},{ }_{1} B_{n}, \cdots,{ }_{8} B_{n}$. Clearly the ${ }_{n} B_{n}$ fill up ${ }_{n} B_{0}$ from the interior for $h=0, \cdots, 8$ (see IV, 10,1). Denote by ${ }_{h} S_{n}$ the surface having the representation $\left[x_{n}(u),{ }_{n} B_{n}\right]$ for $h=0, \cdots, 8, n=0, n>n(I)$. Since the representations $\left[x_{n}(u),{ }_{h} B_{n}\right]$ for $n>n(I)$ are $A C E$ and the essential areas $e A\left({ }_{h} S_{0}\right)$ are finite for $h=0, \cdots, 8$, it follows that (see IV, 6)

4.

$$
e A\left({ }_{h} S_{n}\right)=\int_{n}\left\|\mathcal{F}\left(u,\left[x_{n}, \mathfrak{B}_{n}\right]\right)\right\| d u \quad \text { for } n>n(I)
$$

$$
e A\left({ }_{h} S_{0}\right) \geqq \int_{h_{n} B_{n}^{0}}\left\|\mathcal{F}\left(u,\left[x_{0}, \mathfrak{B}_{0}\right]\right)\right\| d u \quad \text { for } h=0, \cdots, 8 .
$$

From V, 10, 2 it follows that ${ }_{h} S_{n}$ converges to ${ }_{h} S_{0}$ for $h=0, \cdots, 8$, so that (see IV, 5)

$$
\lim \inf e A\left({ }_{h} S_{n}\right) \geqq e A\left({ }_{h} S_{0}\right) \quad \text { for } h=0, \cdots, 8 .
$$

Since straight line segments form the subdivision of $\mathfrak{B}_{n}$ just introduced, one obtains

$$
e A\left(S_{n}\right)=\int_{\mathfrak{B}_{n}^{0}}\left\|\mathcal{F}\left(u,\left[x_{n}, \mathfrak{B}_{n}\right]\right)\right\| d u=\sum_{h=0}^{8} e A\left({ }_{h} S_{n}\right) \quad \text { for } n>n(I) .
$$

Using the preceding relations and $\mathrm{V}, 9,4$, one finds

$$
\lim \sup e A\left(S_{n}\right)=\lim \sup \left[e A\left(S_{n}\right)-\sum_{h=1}^{8} e A\left({ }_{h} S_{n}\right)\right] \leqq \int_{I}\left\|\mathcal{F}\left(u,\left[x_{0}, \mathfrak{B}_{0}\right]\right)\right\| d u \text {. }
$$

In view of relations $2,3,4$, this implies that

5.

$$
\lim u\left(I ;\left[\Psi_{n}, I\right]\right)=u\left(I ;\left[\Psi_{0}, I\right]\right)
$$

for $I$ in $\mathfrak{B}_{0}^{0}$.

Thus, if $\Im$ be any fixed interval in $\mathfrak{B}_{0}^{0}$ and $\mathfrak{F}$ be the class of all intervals $I$ in $\Im$, it is clear from relations 1,5 that the $\left[\psi_{n}, \mathcal{F}\right]$ for $n=0, n>n(\Im)$ satisfy the hypotheses of the general lemma in $\mathrm{V}, \mathbf{5}$. In view of relation 2 , therefore,

$$
\lim \int_{I}\left|\mathcal{F}\left(u,{ }^{i} T_{n}\right)\right| d u=\int_{I}\left|\mathcal{F}\left(u,{ }^{i} T_{0}\right)\right| d u \quad \text { for } I \text { in } \mathfrak{B}_{0}^{0}, i=1,2,3 .
$$

This relation, together with the conditions in $\mathrm{V}, 10$, makes it clear that the hypotheses of the modified closure theorem in II, 14 are fulfilled, for the three sequences of transformations ${ }^{i} T_{n}:\left[{ }^{i} x_{n}(u), \mathfrak{B}_{n}^{0}\right], n=0,1,2, \cdots$, where $i=1$, 2, 3. So ${ }^{i} T_{0}$ is AC $E\left({ }^{i} T_{0}, \mathfrak{B}_{0}^{0}\right)$ for $i=1,2,3$-that is, $\left[x_{0}(u), \mathfrak{B}_{0}\right]$ is AC $E$ (see IV, 1), and the theorem is established.

12. The theorem just proved permits the following addition to the results in IV, 6-9. 
THEOREM. Assume that for a continuous surface $S_{0}$ there exists a sequence of surfaces $S_{n}$ such that $S_{n}$ converges to $S_{0}, e A\left(S_{n}\right)$ converges to eA $\left(S_{0}\right)$ which is finite, and each $S_{n}$ for $n=1,2, \cdots$ has an AC $E$ representation. Then a necessary and sufficient condition that a representation $\left[x_{0}(u), \mathfrak{B}_{0}\right]$ for $S_{0}$ be absolutely continuous $(e A, \mathcal{F})$ is that $\left[x_{0}(u), \mathfrak{B}_{0}\right]$ be AC $E$.

Proof. That the condition is sufficient is already established (see IV, 9). So suppose that $\left[x_{0}(u), \mathfrak{B}_{0}\right]$ is absolutely continuous $(e A, \mathcal{F})$; then (see $\left.I, 8\right)$

$$
e A\left(S_{0}\right)=\int_{\mathfrak{B}_{0}^{0}}\left\|\mathcal{F}\left(u,\left[x_{0}, \mathfrak{B}_{0}\right]\right)\right\| d u .
$$

The $S_{n}$ for $n=0,1,2, \cdots$ thus satisfy the hypotheses of the theorem in $\mathrm{V}$, 9, whence it follows that $\left[x_{0}(u), \mathfrak{B}_{0}\right]$ is AC $E$ :

13. If in the theorem in $\mathrm{V}, 9$, the generalized Jacobians are replaced by the ordinary Jacobians, there results the

TheOREM. Let $S_{n}, n=0,1,2, \cdots$ be a sequence of continuous surfaces satisfying the following conditions:

1. the surfaces $S_{n}$ converge to $S_{0}($ see $I, 1)$;

2. the surface $S_{0}$ has a representation $\left[x_{0}(u), \mathfrak{B}_{0}\right]$ for which the triple $J\left(u,\left[x_{0}, \mathfrak{B}_{0}\right]\right)$ of ordinary Jacobians exists almost everywhere in $\mathfrak{B}_{0}^{0}$, and is summable on $\mathfrak{B}_{0}^{0}($ see IV, 1$)$;

3. the surfaces $S_{n}$ for $n=1,2, \cdots$ have representations which are AC $E$;

4. the essential areas $e A\left(S_{n}\right)$ converge to $\int_{\mathfrak{B}_{0}^{0}}\left\|J\left(u,\left[x_{0}, \mathfrak{B}_{0}\right]\right)\right\| d u$.

Then

5. the representation $\left[x_{0}(u), \mathfrak{B}_{0}\right]$ is $\mathrm{AC} E$;

6. the representation $\left[x_{0}(u), \mathfrak{B}_{0}\right]$ is absolutely continuous $(e A, J)$;

7. the essential variations $\mathrm{eV}\left({ }^{i} S_{n}\right)$ for the projection surfaces ${ }^{i} S_{n}$ converge to $e V\left({ }^{i} S_{0}\right)$ for $i=1,2,3$.

A proof may be made by paralleling the proof for the theorem in $\mathrm{V}, \mathbf{9}$, using the modified closure theorem in II, 15.

14. The preceding theorem permits the following addition to the results in IV, 10-13. A proof is similar to that in V, 12 .

THEOREM. Assume that for a continuous surface $S_{0}$ there exists a sequence of surfaces $S_{n}$ such that $S_{n}$ converges to $S_{0}, e A\left(S_{n}\right)$ converges to eA $\left(S_{0}\right)$ which is finite, and each $S_{n}$ for $n=1,2, \cdots$ has an AC $E$ representation. Then a necessary and sufficient condition that a representation $\left[x_{0}(u), \mathfrak{B}_{0}\right]$ for $S_{0}$, for which the triple $S\left(u,\left[x_{0}, \mathfrak{B}_{0}\right]\right)$ of ordinary Jacobians exists almost everywhere in $\mathfrak{B}_{0}^{0}$, be absolutely continuous $(e A, J)$ is that $\left[x_{0}(u), \mathfrak{B}_{0}\right]$ be AC $E$.

15. CoRollary. A necessary condition that a representation $[x(u), \mathfrak{B}]$ for a continuous surface $S$ be absolutely continuous $(A, J)$, where $A(S)$ is the Lebesgue area of $S$ and $J(u,[x, \mathfrak{B}])$ is the triple of ordinary Jacobians is that 
$[x(u), \mathfrak{B}]$ be AC $E$; if $[x(u), \mathfrak{B}]$ is absolutely continuous $(A, J)$, then the Lebesgue area $A(S)$ equals the essential area $e A(S)$. A sufficient condition that a representation $[x(u), \mathfrak{B}]$ for $S$ be absolutely continuous $(A, J)$ is that $[x(u), \mathfrak{B}]$ be $\mathrm{AC} E$ and $e A(S)=A(S)$.

Proof. The second assertion in this corollary has been established in IV, 14. According to I, 6, 2, there exists a sequence of polyhedra $P_{n}$ which converge to $S$ and for which $A\left(P_{n}\right)$ converges to $A(S)$. Now (see IV, 14) each of the $P_{n}$ has an AC $E$ representation, and $e A\left(P_{n}\right)=A\left(P_{n}\right)$ for $n=1,2, \cdots$. The remainder of the corollary now follows at once from the theorem in $V$, 14.

The results of Rado and Reichelderfer cited in V, 2 are seen to be a special case of this corollary and of the theorem in V, 9 (see II, 15; III, 4, 2; V, 7).

P. Frankin and N. Wiener

\section{BIBLIOGRAPHY}

1. Analytic approximations to topological transformations, Trans. Amer. Math. Soc. vol. 28 (1926) pp. 762-785.

Z. DE GEÖCZE

1. Quadrature des surfaces courbes, Mathematische und naturwissenschaftliche Berichte aus Ungarn vol. 26 (1908) pp. 1-88.

G. H. Hardy, J. E. Litrlewood, G. Polya

1. Inequalities, Cambridge, 1934.

B. VON KEREKJÁRTó

1. Vorlesungen über Topologie, vol. 1, Berlin, 1923.

C. KURATOWSKI

T. RADO

1. Topologie. I, Warsaw-Lw6w, 1933.

1. Sur l'aire des surfaces courbes, Acta Univ. Szeged vol. 3 (1927) pp. 131-169.

2. Über das Flächenmass rektifizierbarer Flächen, Math. Ann. vol. 100 (1928), pp. 445-479.

3. On the problem of Plateau, Ergebnisse der Mathematik und ihrer Grenzgebiete, Berlin, 1933.

4. A lemma on the topological index, Fund. Math. vol. 27 (1936) pp. 212-225.

T. RADO AND P. REICHELDERFER

1. A theory of absolutely continuous transformations in the plane, Trans. Amer. Math. Soc. vol. 49 (1941) pp. 258-307.

2. On convergence in length and convergence in variation, accepted for publication by the Duke Math. J.

S. SAKS

1. Theory of the integral, Warsaw-Lw6w, 1937.

L. TONELLI

1. Sulla quadratura delle superficie, Rendiconti delle sedute della reale accademia nazionale dei Lincei, Classe di Scienze fisiche, matematiche e naturali, vol. 3 (1926) pp. 357-362; pp. 445-450; pp. 633-638.

University of Chicago, Chicago, Ill. 